\title{
Das Schneider-Stipendium des Kriegssekretärs Michael John \\ Ein außergewöhnliches Beispiel privater Studienförderung aus dem vormodernen Leipzig
}

von

MARCEL KORGE

Seit einigen Jahrzehnten erlebt die Forschung zum Stiftungswesen einen spürbaren Aufschwung. Dennoch erfährt ein wichtiger Teilbereich, der sich mit der Studienförderung durch Stipendien beschäftigt und sich im deutschsprachigen Raum den mindestens 4000 bis 6000 Stipendienstiftungen (15. bis 20. Jahrhundert) widmet, in der Wissenschaftsgemeinde weiterhin relativ wenig Aufmerksamkeit. ${ }^{1}$ Stipendienstiftungen bildeten nur einen Teil der Studienförderungsmöglich-

1 Bernhard Ebneth, Stipendium und Promotion. Studienförderung vor und nach der Reformation, in: Rainer Christoph Schwinges (Hg.), Examen, Titel, Promotionen. Akademisches und staatliches Qualifikationswesen vom 13. bis zum 21. Jahrhundert (Veröffentlichungen der Gesellschaft für Universitäts- und Wissenschaftsgeschichte 7), Basel 2007, S. 489-533, hier S. 489, 500; René Franken, Die Kölner Studienstiftungen in der Frühen Neuzeit, in: Jonas Flöter/Christian Ritzi (Hg.), Bildungsmäzenatentum. Privates Handeln - Bürgersinn - kulturelle Kompetenz seit der Frühen Neuzeit (Beiträge zur Historischen Bildungsforschung 33), Köln/Weimar/Wien 2007, S. 73-84, hier S. $76 \mathrm{f}$. Eine treffend knappe Definition des Stiftungsbegriffs findet sich bei Borgolte: „Bei der Stiftung stellt der Geber bekanntlich ein Gut zur Verfügung, das auf Dauer einen von ihm gesetzten Zweck erfüllen soll.“ Michael Borgolte, Die Stiftungsurkunden Heinrichs II. Eine Studie zum Handlungsspielraum des letzten Liudolfingers, in: Karl Rudolf Schnith/Roland Pauler (Hg.), Festschrift für Eduard Hlawitschka zum 65. Geburtstag (Münchener Historische Studien, Abteilung Mittelalterliche Geschichte 5), Kallmünz 1993, S. 231-250, hier S. 232. Zur Etymologie des Begriffs, Stipendium' siehe: Bernhard Ebneth, Stipendienstiftungen in Nürnberg. Eine historische Studie zum Funktionszusammenhang der Ausbildungsförderung für Studenten am Beispiel einer Großstadt (15.-20. Jahrhundert) (Nürnberger Werkstücke zur Stadt- und Landesgeschichte 52), Nürnberg 1994, S. 22-25; Theresa Ratajszczak, Landesherrliche Bildungspolitik und bürgerliches Mäzenatentum. Das Stipendienwesen an der Universität Leipzig 1539-1580 (Beiträge zur Leipziger Universitäts- und Wissenschaftsgeschichte B/14), Leipzig 2009, S. 41 f. Für den vorliegenden Aufsatz soll ein engeres Begriffsverständnis gelten, wonach unter einer Stipendienstiftung jene Stiftung zu verstehen ist, deren Erträge zur Studienförderung von Personen an einer Hochschule angewendet werden sollen. Sachstipendien und Altar- bzw. Messstiftungen werden nicht berücksichtigt. Zur Bedeutung von Altar- und Messstiftungen für die Studienförderung siehe: Eвneth, Nürnberg, S. 20; Ratajszczak, Bildungspolitik, S. 43 f.; Klaus Wriedt, Studienförderung und Studienstiftungen in norddeutschen Städten (14.-16. Jahrhundert), in: Heinz Duchhardt (Hg.), Stadt und Universität (Städteforschung A/33), Köln/Weimar/Wien 1993, S. 33-49, hier S. 39-41. 
keiten, doch gilt es ihre Bedeutung für die Studierenden früherer Jahrhunderte nicht zu unterschätzen. ${ }^{2}$ Ihre Geschichte in Deutschland beginnt zeitgleich mit der Entstehung der ersten Hochschulen im Mittelalter.

Nach ihren Stiftern lassen sich staatlich-öffentliche und nichtstaatlich-private Stipendienstiftungen unterscheiden. In den letzten Jahren kam vorrangig letzterer Gruppe geschichtswissenschaftliches Interesse $\mathrm{zu}^{3}{ }^{3}$

In vielen Fällen überantworteten die staatlichen und privaten Stifter das Stiftungskapital einer Universität, einer Stadt oder deren Unterbehörden zur mündelsicheren Anlage. Die genannten Institutionen hatten vielfach über Präsentations-, Empfangsprüfungs- und Vergaberechte an den Stiftungen teil. Für gewöhnlich lagerten Unterlagen für (Stipendien-)Stiftungen, an denen Universität und/oder Stadt dispositive bzw. administrative Funktionen besaßen, vor allem bei universitären und städtischen Behörden. Die vom Stifter erlassenen Bedingungen privater Stiftungen konnten jedoch auch andere Einrichtungen oder Privatpersonen als Stiftungsorgane vorsehen. Ein geringer Institutionalisierungsgrad, der zum Teil hohe verwaltungstechnische Aufwand und die Einschätzung der Dokumentation privater Stipendienstiftungen als nicht überlieferungswürdig bedingten eine bislang kaum vorgenommene bzw. kaum zu realisierende Analyse dieser Stiftungen. Viele historische Beispiele privater Studienförderung sind heute demnach unbekannt. Wie groß der mutmaßlich erhebliche Anteil jener Stiftungen an der Gesamtheit der Privatstiftungen ist, muss im Dunkeln bleiben. ${ }^{4}$

21871 studierte mindestens jeder vierte Student im deutschsprachigen Raum mithilfe eines oder mehrerer Stipendien. Für die Frühe Neuzeit gehen die Schätzungen deutlich auseinander (zwischen $10 \%$ und mehr als 75 \%). Eвneth, Stipendium (wie Anm. 1), S. 500 f., 519.

3 Oliver Auge, „Zum Nutzen der daselbst studirenden Jugend von gottseligen Herzen gestiftet". Private Stipendien und Studienstiftungen an der Universität Greifswald bis 1945, in: Dirk Alvermann/Karl-Heinz Spieß (Hg.), Universität und Gesellschaft. Festschrift zur 550-Jahrfeier der Universität Greifswald 1456-2006, Bd. 2: Stadt, Region und Staat, Rostock 2006, S. 135-168, hier S. 136.

4 Ebneth, Nürnberg (wie Anm. 1), S. 81; Andreas Gössner, Die Studenten an der Universität Wittenberg. Studien zur Kulturgeschichte des studentischen Alltags und zum Stipendienwesen in der zweiten Hälfte des 16. Jahrhunderts (Arbeiten zur Kirchen- und Theologiegeschichte 9), Leipzig 2003, S. 69 f.; Katrin Löffler, Privatstipendien für Leipziger Studenten im 18. Jahrhundert, in: Detlef Döring (Hg.), Universitätsgeschichte als Landesgeschichte. Die Universität Leipzig in ihren territorialgeschichtlichen Bezügen (Beiträge zur Leipziger Universitäts- und Wissenschaftsgeschichte A/4), Leipzig 2007, S. 339-372, hier S. 352 f. Auch wenn unter den 750 Studierenden der Leipziger Universität um 1800 neben mehr als 200 Nutznießern des Konvikts gegen 150 kurfürstliche Stipendiaten und etwa 100 bis 150 Empfänger von Privatstipendien angenommen werden können, basieren diese Angaben allein auf den bislang nachgewiesenen Stiftungen. Die Gesamtzahl der vormals existenten Stiftungen bleibt unbekannt. Vgl. ebd., S. 353 f.; Alrun Tauché, Staatliches und privates Stiftungswesen an der Universität Leipzig. Das Konvikt im 18. Jahrhundert, in: Flöter/ Ritzi, Bildungsmäzenatentum (wie Anm. 1), S. 177-206, hier S. 202. Zu letzterem Aufsatz vgl. fast inhaltsgleich: Dies., Das Konvikt an der Universität Leipzig im 18. Jahrhundert. Profil, soziale und wirtschaftliche Bedeutung, in: Döring, Universitätsgeschichte als Landesgeschichte, S. 239-259. 
Dies ist umso bedauerlicher, als in den letzten Jahren verstärkt sozialhistorische Fragestellungen in der Stiftungsforschung an Bedeutung gewannen. Insbesondere die Analyse der sozialen Zusammensetzung der spätmittelalterlichen und frühneuzeitlichen Studierendenschaft bzw. Stipendienempfängerschaft wurde in den letzten Jahrzehnten als ein drängendes Desiderat der Universitätshistoriografie erkannt. ${ }^{5}$ Welchen Anteil nahmen die Nachkommen der Adligen, der Geistlichen, der Gelehrten, der Kaufleute an der Studentenschaft ein? Wie stark waren städtische oder kleinstädtisch-dörfliche Mittelschichten vertreten? Konnten sich Angehörige unterer sozialer Schichten über ein Stipendium den Weg an eine der Hochschulen bahnen?6 Eine wichtige Quellengruppe zur Beantwortung dieser Fragen stellen grundsätzlich die Matrikeln der einzelnen Universitäten dar. Leider geben die frühen Immatrikulationseinträge oft nur wenige Hinweise auf die soziale Herkunft der Universitätsverwandten und den elterlichen (in der Regel väterlichen) Berufsstand. Über Namenszusätze, Bemerkungen und Markierungen in den Einschreibebüchern lassen sich zumindest einige soziale Gruppen in adäquatem Maße nachweisen (z. B. Adlige). ${ }^{7}$ Konkretere Differenzierungen bleiben in den Matrikellisten bis ins 19. Jahrhundert die Ausnahme. Häufig nimmt deren Informationsdichte diesbezüglich erst nach $1850 \mathrm{zu}$.

\section{Das Vermächtnis}

Die vorausgegangenen, einführenden Worte gelten nicht zuletzt für die 1409 gegründete Leipziger Universität. Für die Zeit vor dem 19. Jahrhundert ist über die soziale Zusammensetzung ihrer Studierendenschaft wenig, über die ihrer Stipendiatenschaft sehr wenig bekannt. In der Literatur kursieren bis in die Gegenwart

5 Ulrike Denk, Alltag zwischen Studieren und Betteln. Die Kodrei Goldberg, ein studentisches Armenhaus an der Universität Wien, in der Frühen Neuzeit (Schriften des Archivs der Universität Wien 16), Göttingen 2013, S. 100. Für Kitzingen und Nürnberg liegen hervorragende Bearbeitungen dieser Thematik vor: EвNETH, Nürnberg (wie Anm. 1), insbesondere S. 290-294, 308; Martin Riegel, Studienförderung in Kitzingen von der Reformation bis zum Dreißigjährigen Krieg. Stipendienstiftungen - Stipendienwesen - Stipendiaten (Schriften des Stadtarchivs Kitzingen 7), Kitzingen 2006, insbesondere S. 320-328.

6 Erschwerend kommen Abgrenzungsprobleme zwischen den beruflichen bzw. sozialen Schichten hinzu. Siehe z. B.: EBnETH, Nürnberg (wie Anm. 1), S. 149.

7 So in Bezug auf die Leipziger Matrikel: Georg ERLER (Hg.), Die jüngere Matrikel der Universität Leipzig, 1559-1809. Als Personen- und Ortsregister bearbeitet und durch Nachträge aus den Promotionsakten ergänzt, Bd. I: Die Immatrikulationen vom Wintersemester 1559 bis zum Sommersemester 1634, Leipzig 1909, S. XLII-XLVI. Eine umfassende Auswertung der Leipziger Matrikel des 16. und 17. Jahrhunderts erfolgte bereits (WENKE BöHNISCH, Universitäten und Fürstenschulen zwischen Krieg und Frieden. Eine Matrikeluntersuchung zur mitteldeutschen Bildungslandschaft im konfessionellen Zeitalter (1563-1650), Berlin 2013), eine weitere Studie zu den älteren Leipziger Immatrikulationen ist im Entstehen begriffen (Thomas LANG, Der Einzugsbereich der Universität Leipzig im späten Mittelalter 1409-1539). 
hinein noch immer die auf Wilhelm Bruchmüller (1872-1935) zurückgehenden Vorstellungen einer universitas pauperum, einer ,Hochschule der Armen'. Dabei differenzierte bereits Bruchmüller, indem er mehrfach ausführte, dass die Gruppe der Universitätsbesucher in mindestens drei Kategorien zerfiel. Er stellte neben die pauperes jene auf Äußerlichkeiten bedachten adligen oder besitzbürgerlichen "Stutzer" sowie die große Gruppe der „Arbeitsamen“, allerdings sei unter Leipziger Studierenden der Anteil der Habenichtse besonders groß. Besitzlose Studenten würden angezogen durch die sich in der Messe-, Handels-, Buch-, Universitäts- und Bürgerstadt vielfach bietenden Nebenerwerbsmöglichkeiten. Detaillierte Aussagen waren Bruchmüller nicht möglich. ${ }^{8}$ Umso wichtiger sind die Erkenntnisse, die einige jüngere Forschungsarbeiten liefern und die in der umfassenden Jubiläumsausgabe zur Geschichte der Universität Leipzig zusammengeführt wurden. ${ }^{9}$ Dabei wird auf die Bedeutung der Immatrikulationsgebühren für die Analyse der studentischen Sozialstruktur gesondert eingegangen, wobei deren Wert bei realistischer Einschätzung begrenzt bleibt. ${ }^{10}$ Letztlich gelangt man über die Verbindung der Universitätsmatrikel mit anderen lokalen Quellen zu der Er-

8 Wilhelm Bruchmüller, Der Typus des Leipziger Studenten im 18. Jahrhundert, in: NASG 29 (1908), S. 316-323, 335, 337. Fast inhaltsgleich zu diesem Aufsatz auch das 6. Kapitel bei: Ders., Der Leipziger Student 1409-1909, Leipzig 1909 (Nachdruck Langenhagen 2009). Eine erweiterte Variante zur genannten Dreiteilung der Studentenschaft siehe bei: Denk, Alltag (wie Anm. 5), S. 102-107.

9 Geschichte der Universität Leipzig 1409-2009, hrsg. im Auftrag des Rektors der Universität Leipzig Professor Dr. iur. Franz Häuser von der Senatskommission zur Erforschung der Leipziger Universitäts- und Wissenschaftsgeschichte, 5 Bände, Leipzig 2009-2010. Hieraus besonders: EnNo BünZ, Gründung und Entfaltung. Die spätmittelalterliche Universität Leipzig 1409-1539, in: ebd., Bd. 1: Spätes Mittelalter und Frühe Neuzeit 1409-1830/31, Leipzig 2009, S. 17-325, hier S. 142-153. Siehe auch Kirsten Bernhardt, Geselligkeit im Schlafrock. Leipziger Studententumulte im frühen 18. Jahrhundert, in: Kirsten Bernhardt/Barbara Krug-Richter/Ruth-E. Mohrmann (Hg.), Gastlichkeit und Geselligkeit im akademischen Milieu in der Frühen Neuzeit, Münster u. a. 2013, S. 101-112, hier S. 105. Thomas Adam analysierte das Sozialprofil der Leipziger Stipendienbewerber anhand von Akten des Universitätsarchivs, die allerdings aus der zweiten Hälfte des 19. Jahrhunderts stammen. Aus ihnen ergab sich, dass die Väter von etwa 16 Prozent der Stipendienbewerber aus dem Handwerk kamen (Stichproben von 1850, 1860, 1870). Eigene Berechnungen auf der Basis der Angaben von: Thomas Adam, Stipendienstiftungen und der Zugang zu höherer Bildung in Deutschland von 1800 bis 1960 (Pallas Athene. Beiträge zur Universitätsund Wissenschaftsgeschichte 28), Stuttgart 2008, S. 130-134. Unter den Studenten der Universität Halle besaßen Handwerkersöhne zwischen 1761 und 1778 einen Anteil von zwölf Prozent. Hans-Ulrich Wehler, Deutsche Gesellschaftsgeschichte, Bd. 1: Vom Feudalismus des Alten Reiches bis zur Defensiven Modernisierung der Reformära 1700-1815, München 31996, S. 298.

10 Bünz, Gründung (wie Anm. 9), S. 151-153; Manfred Rudersdorf, Weichenstellung für die Neuzeit. Die Universität Leipzig zwischen Reformation und Dreißigjährigem Krieg 1539-1648/1660, in: Geschichte der Universität Leipzig 1409-2009 (wie Anm. 9), Bd. 1: Spätes Mittelalter und Frühe Neuzeit 1409-1830/31, Leipzig 2009, S. 327-515, hier S. 506. Vgl. Alexander Sembdner, Stadt und Universität Leipzig im späten Mittelalter (Beiträge zur Leipziger Universitäts- und Wissenschaftsgeschichte B/17), Leipzig 2010, S. 66 f. 
kenntnis, dass insbesondere im Spätmittelalter die meisten Leipziger Studenten klein- bzw. mittelstädtischer bürgerlicher Herkunft waren und der Anteil adliger Studenten nach der Reformation vorübergehend zunahm. ${ }^{11}$ Ein anderer jüngerer Beitrag zur Universitätsgeschichte des 18. Jahrhunderts geht ebenfalls der Frage der sozialen Verortung von Universitätsverwandten nach und stützt sich dabei auf 35 Nachlassakten von Leipziger Studierenden, deren Mehrzahl akademisch gebildeten Familien entstammte. Nur drei dieser 35 Studenten kamen aus dem Handwerksmilieu. Ein Stipendienbezug konnte aber nicht belegt werden. ${ }^{12}$

Für die Alma mater Lipsiensis konnten vor wenigen Jahren durch einige Studien, nicht zuletzt durch das damals bevorstehende 600-jährige Universitätsjubiläum angeregt, neue instruktive Erkenntnisse zum Stipendienwesen präsentiert werden, ${ }^{13}$ wenngleich auch hier ein Teil der privaten Stiftungen ohne ausreichend intensive Untersuchung bleiben musste. Dabei bestanden viele der privaten Stiftungen vermutlich jahrhundertelang. Eine solche langlebige, bislang nahezu unbekannte private Stipendienstiftung wird auf den folgenden Seiten vorgestellt. Durch Archivrecherchen konnte sie als Teil eines größeren testamentarischen Vermächtnisses nachgewiesen werden, wogegen sie in den für die potenziellen Stipendienempfänger herausgegebenen offiziellen Auflistungen und Sammlungen nicht auftaucht. ${ }^{14}$ Selbst das umfangreiche Stiftungsbuch von Geffcken und Tykocinski nennt nur zwei Teilstiftungen des wesentlich umfangreicheren Vermächtnisses.

11 Bünz, Gründung (wie Anm. 9), S. 148, 150; Rudersdorf, Weichenstellung (wie Anm. 10), S. 506. Vgl. Detlef DöRIng, Anfänge der modernen Wissenschaften. Die Universität Leipzig vom Zeitalter der Aufklärung bis zur Universitätsreform 1650 1830/31, in: Geschichte der Universität Leipzig 1409-2009 (wie Anm. 9), Bd. 1: Spätes Mittelalter und Frühe Neuzeit 1409-1830/31, Leipzig 2009, S. 517-771, hier S. 598.

12 Anja Pohl, Studentische Lebensführung im 18. Jahrhundert. Erkenntnisse aus Nachlaßakten verstorbener Studenten, in: Döring, Universitätsgeschichte als Landesgeschichte (wie Anm. 4), S. 205-237, hier S. 208, 216, 219.

13 Beispielsweise: Ratajszczak, Bildungspolitik (wie Anm. 1); Tauché, Stiftungswesen (wie Anm. 4). Vgl. auch BüNz, Gründung (wie Anm. 9), S. 153 f. Die älteste Leipziger Stipendienstiftung, welche zugleich zu den Privatstiftungen gerechnet werden kann, datiert aus dem Jahr 1460 und ist abgedruckt in: Codex diplomaticus Saxoniae regiae, Zweiter Hauptteil, Bd. 11: Urkundenbuch der Universität Leipzig von 1409 bis 1555, hrsg. von Bruno Stübel, Leipzig 1879, S. 140-142, Nr. 121. Vgl. Heinrich Geffcken/ Chaim Trкocinski, Stiftungsbuch der Stadt Leipzig, Leipzig 1905, S. XVI; Ratajszczak, Bildungspolitik (wie Anm. 1), S. 94; Chaim Tykocinski, Die Stiftungen der Stadt Leipzig vor der Reformation, in: Wissenschaftliche Beilage der Leipziger Zeitung 1903, Nr. 121, 13. Oktober, S. 487.

14 Max Baumgart, Die Stipendien und Stiftungen (Convicte, Freitische u. s. w.) zu Gunsten der Studirenden an allen Universitäten des deutschen Reichs nebst den Statuten und Bedingungen für die Bewerbung und den Vorschriften über die Stundung resp. den Erlass des Collegienhonorars, Berlin 1885; CARL Christian Canis Gretschel, Die Universität Leipzig in der Vergangenheit und Gegenwart, Dresden 1830, Stipendientabellen im Anhang; Moritz Meltzer, Verzeichniß der Stipendien und Beneficien, welche ausschließlich oder doch event. für Studirende an der Universität Leipzig fundirt sind, Leipzig ${ }^{3} 1896$. 
Die besagte Stipendienstiftung fehlt jedenfalls. ${ }^{15}$ Sie fand in den städtischen und universitären Quellen ebenfalls kaum Niederschlag, da sie durch ihre spezielle Stiftungsstruktur administrativ ausschließlich in den Händen einer anderen Institution lag.

Nachdem die reiche Handels- und Messestadt Leipzig im Dreißigjährigen Krieg mehrfach und von verschiedenen Seiten, mal durch kaiserliche, mal durch schwedische Truppen, belagert, erobert und geplündert worden war, übernahmen die Schweden Ende 1642 die Kontrolle über die Stadt. Die Besatzungszeit sollte über den Westfälischen Frieden hinaus bis zum Sommer 1650 dauern. ${ }^{16}$

Noch mitten im Krieg verfasste am 13. Dezember 1644 mit zittriger Hand ein schwerkranker Mann einen Codicill17 in seiner Wohnung im Leipziger Ratsmarstall. ${ }^{18}$ Schon beträchtlich von einem lebensgefährlichen Leiden gezeichnet, lud er zehn Tage später den angesehenen Stadtrichter Jacob Merzner, dessen Assessor Sebastian Drewer, den Gerichtsnotar Matthaeo Schilling sowie weitere Personen als Zeugen zu sich und übergab ihnen das Dokument in einem versiegelten Kuvert zur gerichtlichen Verwahrung. Vor den Augen der Anwesenden unterschrieb der 39-Jährige in seinem Krankenbett liegend den Umschlag und bat sich einige mündliche Ergänzungen aus, die ihm gestattet wurden. So hatte er seine Patentochter, die Tochter des Tischlers Carll Hartman, im Testament vergessen, was er nun korrigierte. Die wesentlichsten Festlegungen des in mehrere Punkte unterteilten Codicills blieben aber von den Veränderungen unberührt.

Bei dem überlieferten Schriftstück handelte es sich nicht um die erste, aber doch, wenn man von den genannten kleinen Ergänzungen absieht, um die letzte Fassung des testamentarischen Willens, denn der Testator, der den Namen Michael John trug, ${ }^{19}$ sollte nur noch wenige Tage leben. Obwohl er noch keine 40 Jahre

15 Geffcken, Stiftungsbuch (wie Anm. 13), S. 144 f., Nr. 255.

$16 \mathrm{Zu}$ den Auswirkungen auf Stadt und Universität siehe: Alexander Zirr, Stadt und Universität Leipzig im Dreißigjährigen Krieg, in: Detlef Döring (Hg.), Stadt und Universität Leipzig. Beiträge zu einer 600-jährigen wechselvollen Geschichte (Quellen und Forschungen zur Geschichte der Stadt Leipzig 1), Leipzig 2010, S. 145-166; Ders., Universität und fremde Besatzung. Die ,Alma mater Lipsiensis' während der schwedischen Herrschaft über Leipzig 1642 bis 1650, in: Militär und Gesellschaft in der frühen Neuzeit 15 (2011), H. 1, S. 18-40. Zur Charakterisierung des Dreißigjährigen Krieges als deutliche Zäsur für die Entwicklung des Stiftungswesens siehe: EBNETH, Nürnberg (wie Anm. 1), S. 55; Ders., Stipendium (wie Anm. 1), S. 519.

17 Der besiegelte Codicill, auf den sich viele der folgenden Bemerkungen beziehen, ist im Original zu finden in: Stadtarchiv Leipzig (im Folgenden: StadtA Leipzig), Richterstube, Testamente Rep. V, Paket 71, Nr. 2.

18 Seit 1575 befand sich der Ratsmarstall am Neumarkt an der Ecke Peterskirchhof im Petersviertel. ERnst Müller, Häuserbuch zum Nienborgschen Atlas. Description über die Grund-Legung und in richtigen Abriß gebrachte berühmte Handels-Stadt Leipzig (Quellen und Forschungen zur sächsischen Geschichte 11), Berlin 1997, S. 29, Nr. 193.

19 In verschiedenen Quellen wurde er auch Michel John oder Michael Jahn genannt. Verwechslungsgefahr besteht mit einem Zeitgenossen, dem Leipziger Academicus und Stublschreiber Michel John, der - vielleicht in Abgrenzung zu dem Sekretarius und 
zählte, hatte sich John in diesen gefährlichen Kriegs- und Seuchenzeiten schon mehrfach mit der Möglichkeit seines baldigen Ablebens beschäftigt, denn er habe, wie es in der knappen Einleitung des Codicills heißt, seinen letzten Willen zu vntterschiedenen mablen auffgesetzt ond durch gutte freunde vollzogen. [...] Nachdem aber dem Menschen allerband Zufälle, die seinen sin vnndt meinung endern, $z u$ handen stoßen, hab ich auch solches erfabren müßen, worauf nochmals eine Überarbeitung erfolgte.

Nach den wenigen einleitenden Worten schlossen sich auf gut zwei Seiten die weiteren Ausführungen zur Verwendung des Johnschen Nachlasses an. Der Aussteller des Testaments verfügte eine Teilung seiner Besitztümer unter mehr als ein Dutzend Privatpersonen und Institutionen, was damals nicht unüblich war. So sollten die Anverwandten, die Paten und der Beichtvater Geldbeträge nach Ableben des Testierenden erhalten. Außerdem setzte John verschiedene Legate auf. Dem Leipziger Hospital St. Jacob (Lazareth) sollten 1600 Reichstaler zustehen, damitt die krancken so viel möglich gewarttet können werden. Das Hauptkapital zu diesem Zweck aufzubrauchen, war nicht vorgesehen. Vielmehr sei dieses sicher und fruchtbringend anzulegen, damit die anfallenden Zinsen fur arme doch vielleicht bürgerliche leutte verwendet werden konnten. Ein weiteres Legat vermachte John der Leipziger Thomasschule. ${ }^{20}$

Die entscheidende Passage zur Stipendienstiftung formulierte John als Punkt Acht des Codicills: Dem Schneider handwerck legir ich fünffhundert Rhlr. [d. h. Reichstaler] Capital, davon sollen die Zinßen, alß $30 \mathrm{Rh}$. [d. h. Reichstaler] zum wenigsten, zu einen stipendio angewendet ond einem Schneiders Sobn, der studirt, $3 \mathrm{Jahr}$ lang zu beßer Fortsetzung seines studirens gereichet werden: studirte keiner nicht, so soll jäbrlich die selbige Zinße vntter arme meister ond wittwen des bandwercks außgetheilet werden.

Bevor sich dieser bemerkenswerten Stipendienstiftung en détail zugewandt wird, soll die Stifterpersönlichkeit näher betrachtet werden.

\section{Der Stifter}

Wir wissen wenig über Michael John, aber doch mehr als über die meisten seiner Zeitgenossen. Zu Lebzeiten war Michael John in die Gesellschaft der kursächsischen Messe- und Universitätsstadt wohl gut integriert, denn er vermachte nicht nur zahlreichen Leipziger Einrichtungen und Privatpersonen verschiedene Geld-

Stifter John - sonst der kleine genant wurde. StadtA Leipzig, Ratsleichenbuch Nr. 16 (1672-1680), fol. 11r.

20 Auf diese Teilstiftung, die bekannter als die Stipendienstiftung ist, wird u. a. verwiesen in: StadtA Leipzig, Ratsbuch 133 (1684-1685), fol. 34v-38v; GefFCKEn, Stiftungsbuch (wie Anm. 13), S. 144 f., Nr. 255; Отто Kaemmel, Geschichte des Leipziger Schulwesens vom Anfange des 13. bis gegen die Mitte des 19. Jahrhunderts (1214-1846), Leipzig/Berlin 1909, S. 57. 
beträge, Sachgüter und Legate, sondern war auch Patenkind von drei Leipziger Bürgern. Michael John kam am 24. August 1605 zur Welt und wurde am Folgetag in der Pfarrkirche St. Nicolai getauft. Als Eltern werden im Taufbuch der ehrsame Leipziger Bürger Christoff Junge (später John, seltener Jahn genannt) und seine Ehefrau Katharina angegeben. ${ }^{21}$

Im Codicill ist bezüglich der 1644 noch lebenden Familienangehörigen neben der Stiefmutter und zwei Stiefbrüdern von einem leiblichen Bruder namens Christoph John die Rede. ${ }^{22}$ Letzterer wurde fast sechs Jahre nach Michael geboren. ${ }^{23}$ Auffällig ist, dass der Bruder im Gegensatz zu der Stiefmutter und den Stiefbrüdern testamentarisch nicht bedacht wurde, wenngleich die familiären $\mathrm{Zu}$ wendungen ohnehin relativ gering ausfielen (nur je ein- bis zweihundert Taler). Sowohl die leiblichen Eltern als auch mehrere Geschwister lebten bei Aufsetzung des Testaments nicht mehr. Die Mutter verlor Michael im Alter von etwa sieben Jahren, kurz nach der Geburt seines Bruders Christoph. Der Vater heiratete bald darauf neu, überlebte auch seine zweite Ehefrau und mehrere seiner Kinder. Er nahm sich 1627 Regina Voitlenderin, Tochter eines Böttchers, zur Frau und starb 1636. ${ }^{24} \mathrm{Zu}$ Ehren des Vaters und zur Erinnerung an ihn sollte nach dem letzten Willen Michael Johns ein Epitaph oder eine schöne Taffel mitt gemäblde oder schrifft gefertigt und in der Pfarrkirche St. Thomas aufgehängt werden.

Viele weitere Details gibt der Codicill preis. Als Michael John Ende 1644 kränklich in seiner Wohnung im Marstall lag, war er von einer gewissen Frau Martha Lufft, der Ehefrau des Ratsbuchhalters Samuel Lufft, ${ }^{25}$ mit einer trewen mütterlichen Warttung umsorgt worden. Doch trotz der Bemühungen seiner Freunde und Verwandten, der Wärterin Lufft und der Leipziger Medizinerschaft sollte John das neue Jahr nicht mehr erleben. Er starb am 26. Dezember 1644. Sein

21 Kirchliches Archiv Leipzig (im Folgenden: KA Leipzig), Taufbuch St. Nicolai 16011606, S. 330; SAlomon STEPNER, Inscriptiones Lipsienses locorum publicorum academicorum pariter ac senatoriorum memorabiles, Leipzig 1675, S. 169, Nr. 718; S. 76, Nr. 248.

22 In den Leipziger Ratsleichenbüchern finden sich in den Folgejahren zwei Einträge, die jeweils auf einen Christoph John verweisen. Zum einen starb bereits im Juli 1645 ein Junggeselle dieses Namens, zum anderen verweist ein weiterer Eintrag auf einen zwischen 1668 und 1682 verstorbenen Bürger und Familienvater. Es konnte nicht geklärt werden, ob sich hinter einem der Einträge der Bruder des Stifters verbarg. StadtA Leipzig, Ratsleichenbuch Nr. 13 (1643-1647), S. 145; Nr. 17 (1680-1689), fol. 138v.

KA Leipzig, Taufbuch St. Thomas 1605-1625, fol. 40r.

24 KA Leipzig, Traubuch St. Nicolai 1609-1623, S. 74; 1624-1646, S. 74; StadtA Leipzig, Ratsleichenbuch Nr. 7 (1625-1627), fol. 81r [inhaltsgleicher Eintrag: Nr. 8 (1626-1631), fol. 25r]; Nr. 10 (1633-1638), fol. 102v; Nr. 13 (1643-1647), S. 46; STEPNER, Inscriptiones (wie Anm. 21), S. 76, Nr. 248. Im Ratsleichenbuch, in welchem das Begräbnis der ersten Stiefmutter von Michael und Christoph John junior verzeichnet wurde, ist vermutlich ein Schreibfehler enthalten. Irrtümlich wird hier von Sabinen statt von Reginen John (geborene Apel) gesprochen. Der Witwer heiratete nach Ablauf des Trauerjahres erneut.

25 KA Leipzig, Taufbuch St. Nicolai 1627-1643, S. 194; Traubuch St. Thomas 1609-1646, fol. 89r. 
Testament wurde auf Ansuchen seines Bruders Christoph einen Monat später auf der Ratsstube publiziert.

Von Johns Begräbnis zeugt ein Eintrag im Leipziger Ratsleichenbuch. ${ }^{26}$ Hier wird der Verstorbene zugleich mit den Zusätzen Junger geselle, Fürstl. Weymarischer geheimbter Secretarius und J. U. Canditat. vorgestellt. Aus diesem Eintrag konnten unter Zuhilfenahme weiterer Quellen wertvolle Informationen gewonnen werden:

Die Bezeichnung Junger geselle war kein Indiz dafür, dass Michael John in besonders jungen Jahren verstarb, sondern ein Hinweis auf seinen ledigen Familienstand. Er war zeitlebens Junggeselle. Viele Jahre stand er als geheimter- und Kriegs-Secretarius in den Diensten Herzog Bernhards von Sachsen-Weimar (16041639), ${ }^{27}$ eines klugen Feldherrn und frühen Verbündeten des Schwedenkönigs Gustav II. Adolf (1594-1632). Nach dem Tod Gustav Adolfs kühlte das Verhältnis zu den Schweden merklich ab, sodass Feldherr Bernhard Verhandlungen mit Frankreich aufnahm und in dessen Dienste trat. An den Unterredungen in Paris nahm Michael John aktiv teil und berichtete seinem Herrn. Er blieb bis zum Tode Bernhards in dessen Diensten. Diese Treue wurde vom Herzog mit einem Legat über 3000 Reichstaler belohnt. ${ }^{28}$ Warum der secretarius John 1644 in Leipzig weilte, ist unbekannt. Ein Teil der Regimenter Herzog Bernhards ging nach dessen Tod zu den Schweden über. Möglich, dass Michael John mit diesen Truppen zog und so ins ab 1642 schwedisch besetzte Leipzig gelangte. Vermutlich trieben ihn mehrere Beweggründe hierher. Die Stadt war ihm als Geburts- und Heimatstadt vertraut. In jungen Jahren hatte er an der Alma mater Lipsiensis studiert und auch später pflegte er seine persönlichen Kontakte. Vermutlich aus der Studienzeit rührte seine Verbundenheit zur hiesigen Universität, welche für die Entstehung der Stipendienstiftung natürlich förderlich war. ${ }^{29} \mathrm{Ob}$ er damals, ein Inskriptions-

26 StadtA Leipzig, Ratsleichenbuch Nr. 13 (1643-1647), S. 117. Anders als üblich wurden die Sterberegister in Leipzig seit dem 16. Jahrhundert nicht in den städtischen Kirchgemeinden, sondern bei der Stadt (Rats-Leichenschreiberei) geführt.

27 Stepner, Inscriptiones (wie Anm. 21), S. 76, Nr. 248; S. 169, Nr. 718.

28 August von Gonzenbach, Der General Hans Ludwig von Erlach von Castelen. Ein Lebens- und Charakterbild aus den Zeiten des dreißigjährigen Kriegs, Teil I, Bern 1880, S. 128; Reinhard Hildebrandt (Hg.), Quellen und Regesten zu den Augsburger Handelshäusern Paler und Rehlinger 1539-1642. Wirtschaft und Politik im 16./17. Jahrhundert, Teil 2: 1624-1642 (Deutsche Handelsakten des Mittelalters und der Neuzeit XIX, 2), Stuttgart 2004, S. 218 f., Nr. 527; S. 239, Nr. 558.

29 Ob dieses Phänomen für das 17. Jahrhundert typisch oder atypisch war, gilt es noch zu beweisen. Für die Zeit vor 1580 liegt der Nachweis schon vor: Die Leipziger Stipendienstifter vor Einführung der Reformation hatten zu etwa drei Viertel in Leipzig studiert. Nach der Reformation (und bis 1580) konnten dagegen nur einzelne Stifter nachgewiesen werden, die sich hier in die Matrikel eingetragen hatten, wobei aber der Anteil der stiftenden Frauen deutlich angestiegen war. Im Vergleich dazu besuchten im 16. Jahrhundert im katholisch bleibenden Ingolstadt fast alle Stipendienstifter die Ingolstädter hohe Schule. Ratajszczak, Bildungspolitik (wie Anm. 1), S. 105, 132; HEINZ JÜRgEN Real, Die privaten Stipendienstiftungen der Universität Ingolstadt im ersten Jahrhundert ihres Bestehens (Ludovico Maximilianea 4), Berlin 1972, S. 123. 
eintrag als iniurati ist in den Universitätsmatrikeln für das Sommersemester 1616 und die Ablegung des Gehorsamsversprechens ist für das dritte Rektorat von Wilhelm Schmuck (1575-1634) im Sommersemester 1624 verzeichnet, ${ }^{30}$ selbst ein Stipendium genoss, wissen wir nicht. Dieses könnte ihm die Sinnhaftigkeit einer solchen Studienförderung vor Augen geführt haben. Motive wie der ,gemeine Nutz' für die Vaterstadt oder ein Gefühl sozialer Verpflichtung für die weniger Begünstigten spielten vermutlich ebenfalls eine Rolle.

Der dritte Zusatz J. U. Canditat. verweist auf einen akademischen Kandidatenstatus im Fachbereich der Rechtswissenschaft, wobei die Abkürzung J. U. für iuris utriusque (d. h. beiderlei Rechte, also weltliches und kirchliches Recht) steht. Somit hatte John die erste akademische Prüfung zum baccalaureus an der juristischen Fakultät erfolgreich absolviert und war befähigt, den Grad eines doctor iuris zu erwerben. ${ }^{31}$ Für seine Anstellung bei dem herzoglich weimarischen Heerführer Bernhard bedurfte es allerdings dieses Grades nicht, sodass er vermutlich bald nach 1624 die Hochschule wieder verließ. ${ }^{32}$ Ein weiteres Interessengebiet des baccalaureus und Schreibers John geht aus seinem Testament hervor. Darin bedachte er seine Krankenwärterin Martha Lufft unter anderem mit einer Anzahl an Büchern, darunter Coleri haußbuch, ${ }^{33}$ krentter buch, Wirsing artzeney buch, ${ }^{34}$ H[errn] Arnds Psalmen ${ }^{35}$ vnd andere geistliche teutsche bücher. Aus dem Besitz geistlicher Bücher auf eine ausgeprägte Religiosität Johns schließen zu wollen, wäre sicher vermessen. Daran ändert der Umstand nichts, dass Johns Beichtvater immerhin mit 50 Reichstalern testamentarisch bedacht wurde. Vielmehr fehlte ein Legat oder eine Schenkung zugunsten einer kirchlichen Einrichtung, wogegen ,soziale Institutionen` wie das Lazarett, der Gotteskasten, die Schülerschaft zu St. Thomas oder allgemein die armen leutte von den Verfügungen profitierten. Das Vorhandensein medizinischer Literatur erlaubt dagegen die Vermutung, dass John zumindest größeres Interesse an diesem Wissenszweig besaß und vielleicht sogar an der Leipziger Universität die eine oder andere Vorlesung hierzu besucht hatte.

30 Erler, Matrikel I (wie Anm. 7), S. 210. Zu Michaels Bruder Christoph John findet sich ein Inskriptionseintrag für das Sommersemester 1623. Ebd., S. 209.

31 Ewald Horn, Die Disputationen und Promotionen an den Deutschen Universitäten vornehmlich seit dem 16. Jahrhundert. Mit einem Anhang enthaltend ein Verzeichnis aller ehemaligen und gegenwärtigen deutschen Universitäten (Beiheft zum Centralblatt für Bibliothekswesen 11), Leipzig 1893, S. 14.

32 Dass John wie im Leipziger Stiftungsbuch zu lesen, den juristischen Doktorgrad erworben habe, konnte nicht eruiert werden. Eventuell wurde der Eintrag bei Erler falsch gedeutet, da hier von einem $D r$. jur. zwar die Rede war, dieser Hinweis sich aber nicht auf John, sondern auf den Rektor Wilhelm Schmuck bezog. Vgl. Geffcken, Stiftungsbuch (wie Anm. 13), S. 144 f., Nr. 255.

33 Johannes Coler (1566-1639), protestantischer Pfarrer. Seine „Oeconomia oder Hausbuch“ umfasst sechs Teile und erschien in einer ersten Auflage zwischen 1593 und 1601.

34 Christoph Wirsung (1500-1571), Augsburger und ab 1562 Heidelberger Arzt und Apotheker. Wirsungs „Artzney Buch“ stellt eine umfangreiche Rezeptsammlung dar, die erstmals 1568 veröffentlicht wurde.

35 Johann Arnd(t) (1555-1621), Theologe der Nachreformation. 
Auch schien John musisch begabt gewesen zu sein, vermachte er doch gleich mehrere Musikinstrumente, darunter ein Spinet ond Clavierdium sowie seine gutte Lautte.

\section{Die Stipendienstiftung}

Kommen wir zurück zur Stipendienstiftung, die nur einen Bruchteil des Johnschen Vermächtnisses (gerade 500 von über 4000 Reichstalern) ausmachte. Will man die Stipendienstiftung den in der Forschung derzeit aktuellen Typologien zuordnen, kommt man zu folgenden Ergebnissen:

Bezüglich des Empfängerkreises ist die Stiftung nach einer Einteilung von Thomas Adam den offenen Stipendienstiftungen zuzurechnen. Dominierten im späten Mittelalter und in der Frühen Neuzeit neben ortsgebundenen vor allem Familienstiftungen bzw. gemischte Stiftungen, die die Angehörigen des Stifters begünstigten, handelte es sich bei der vorliegenden um eine Stiftung, die einer bestimmten sozialen Personengruppe zukam. ${ }^{36}$ Wenngleich eine solche Ausrichtung auf einen spezifischen sozialen bzw. beruflichen Stand bis in die Neuzeit nicht zu den gebräuchlichsten Charakteristika von Stipendienstiftungen zählte, gab es insbesondere unter Geistlichen, Professoren und Ratsherren doch auch jene, die entsprechende Stiftungen für Pfarrers-, Gelehrten- und Ratsherrensöhne errichteten. ${ }^{37}$ Deutlich seltener waren Stipendien, die bevorzugt oder ausschließlich Söhnen aus dem Handwerk oder gar einem einzelnen Gewerbe zur Verfügung standen. Aus dem frühneuzeitlichen Leipzig ist diesbezüglich nur die Stiftung des im Jahr 1741 verstorbenen Hofrats und Advokaten Dr. Johann August Hölzel bekannt. Doch selbst diese Stiftung, welche zwei Stipendien beinhaltete, war primär mit einem Vorrecht für die Söhne der Familie Hölzel verbunden. Nachrangig hatte der Stifter zusätzlich bestimmt, dass, wenn lediglich ein oder kein Familienmitglied in Leipzig studieren würde, ein oder zwei Leipziger bzw. Annaberger Bür-

36 AdAm, Stipendienstiftungen (wie Anm. 9), S. 28. Die Kategorie des väterlichen Berufsstandes als Typologisierungskriterium wird nicht in allen neueren Studien genutzt, wohl aber bei: Auge, Zum Nutzen (wie Anm. 3), S. 151. Auf diesen Beitrag bezieht sich auch: Simon Huemer, Studienstiftungen an der Christian-Albrechts-Universität zu Kiel (1665-1923). Private Bildungsförderung zwischen Stiftungsnorm und Stiftungswirklichkeit (Kieler Werkstücke A/34), Frankfurt a. M. 2013, S. 24, Anm. 46. Dagegen bildeten Familienstiftungen in reiner oder gemischter Form in Greifswald, Ingolstadt, Leipzig (nach der Reformation), Nürnberg, Wien und Wittenberg die wichtigste Gruppe an Stipendienstiftungen, während an den Universitäten Leipzig (vor der Reformation) und Kiel (17.-20. Jahrhundert) ortsgebundene und freie Stipendien dominierten. Auge, Zum Nutzen (wie Anm. 3), S. 151; Denk, Alltag (wie Anm. 5), S. 165; Ebneth, Nürnberg (wie Anm. 1), S. 171-180; Gössner, Wittenberg (wie Anm. 4), S. 91, 101, 164; Huemer, Kiel, S. 33; Löffler, Privatstipendien (wie Anm. 4), S. 358; Ratajszczak, Bildungspolitik (wie Anm. 1), S. 108, 138; Real, Ingolstadt (wie Anm. 29), S. 109.

37 Leipziger Beispiele in: Baumgart, Stipendien (wie Anm. 14), S. 516 f., $526,529$. 
ger- und Handwerksmeistersöhne in den Genuss jährlicher Zuwendungen kommen sollten. ${ }^{38}$

Betrachtet man die Stifterseite, stößt man auf eine auffällig niedrige Anzahl bekannter Stipendienstiftungen aus dem Bereich des Handwerks. Dies war zum einen bedingt durch die die Überlieferung erheblich beeinflussenden Verwaltungsmodalitäten. Die Administration bei Stiftungen, die durch oder für Handwerksorganisationen ins Leben gerufen wurden, oblag in vielen Fällen nicht (allein) städtischen oder universitären Einrichtungen, sondern konnte beispielsweise den Vorstehern (Obermeistern, Ältesten) des Handwerks überantwortet werden. ${ }^{39}$ Hierbei fand die Dokumentation zu Stipendienbewerbung und Stipendienvergabe oft nicht den Weg in die kommunalen oder universitären Archiveinrichtungen.

Relevant für die wenigen bekannten Handwerksstipendien ist zum anderen die soziale Zusammensetzung des potenziellen Stifterkreises. Stipendienstiftungen stammten selten aus dem Handwerksmilieu. Zwar gab es einige reichere Meister bzw. Meisterwitwen, die als Stifter und Stifterinnen auftraten, und Handwerksorganisationen errichteten als Korporationen durchaus diverse Stiftungen, doch diese dienten im Regelfall anderen Zwecken. Vielfach standen religiöse oder sozial-karitative Anliegen im Vordergrund. ${ }^{40}$ Aufgrund der häufigen Berufs- bzw. Werkstattübernahmen durch Handwerkersöhne oder Gesellen, die eine Meistertochter heirateten, und der oft schwierigen finanziellen Verhältnisse, lag es nahe, die Allgemeinbildung dieser Personen zu fördern ${ }^{41}$ bzw. den finanziellen Auf-

38 Ebd., S. 518; GeffCKen, Stiftungsbuch (wie Anm. 13), S. 256, Nr. 421; Meltzer, Verzeichniß (wie Anm. 14), S. 78 f.

39 Евnетн, Nürnberg (wie Anm. 1), S. 166-169.

40 Zahlreiche Leipziger Beispiele für einzelne stiftende Handwerksmeister und Meisterwitwen in: StadtA Leipzig, Stift. I Nr. 80, Vol. I, fol. 18r, 26v; Stift. IV Nr. 2; Geffecken, Stiftungsbuch (wie Anm. 13); Marcel Korge, Kollektive Sicherung bei Krankheit und Tod. Fallstudien zum frühneuzeitlichen Zunfthandwerk in städtischen Zentren Sachsens (Chemnitz, Dresden, Leipzig und Zwickau) (Studien zur Gewerbe- und Handelsgeschichte 33), Stuttgart 2013, S. 229, 433; Walter LAnge, Das erste Halbjahrtausend der Kürschner-Innung zu Leipzig 1423-1923, Leipzig 1925, S. 11-14, 25. Deutlich seltener sind kollektive Stiftungen der Handwerkszünfte oder Gesellenorganisationen überliefert, da diese Gemeinschaften die Stiftungsverwaltung vermutlich nach Möglichkeit in ihren Händen belassen wollten, was wiederum die heutige Forschung vor Überlieferungsprobleme stellt. Einzelne Leipziger Beispiele bei: GefFcken, Stiftungsbuch (wie Anm. 13), S. 27, Nr. 34; S. 46, Nr. 65; S. 55, Nr. 77; S. 68, Nr. 116; Korge, Kollektive Sicherung, S. 229, 231, 305.

41 Die zunehmend wichtiger werdende Allgemeinbildung im Handwerksbereich betont z. B. Rainer S. Elkar, Feder, Tinte und Papier - ungebrauchte Werkzeuge im alten Handwerk?, in: Hans-Jürgen Gerhard (Hg.), Struktur und Dimension. Festschrift für Karl Heinrich Kaufhold zum 65. Geburtstag, Bd. 1: Mittelalter und Frühe Neuzeit (Vierteljahrschrift für Sozial- und Wirtschaftsgeschichte, Beihefte 132), Stuttgart 1997, S. 274-297. Zur schulischen Ausbildung von Handwerkersöhnen an Winkelschulen siehe Thomas Töpfer, Schulwesen und städtische Gesellschaft. Grundprobleme der Bildungsgeschichte des 18. Jahrhunderts am Beispiel Leipzigs, in: Historisches Jahrbuch 127 (2007), S. 175-207, besonders S. 191 f.; Ders., Die „Freyheit“ der Kinder. Territoriale 
wand bei Antritt einer Handwerkslehre, bei der Heirat (möglichst innerhalb des Handwerks) oder bei der Erlangung des Meisterrechts zu verringern. ${ }^{42}$

Somit ist die Zahl vormoderner Stipendienstiftungen aus dem Handwerk äußerst überschaubar. ${ }^{43}$ Nach einer jüngeren Studie zu den Nürnberger Stipendienverhältnissen hätten die wenigen Handwerks-Stipendienstiftungen in der Regel eine bevorzugte Förderung von Söhnen des jeweiligen Gewerbes vorgesehen. ${ }^{44}$ Für Leipzig bestätigt sich diese Einschätzung nicht. Unter den hier bis zur Mitte des 19. Jahrhunderts errichteten Stipendienstiftungen stammten gerade einmal zwei aus Handwerkerkreisen und in beiden Fällen verfügten Bäckermeisterwitwen testamentarisch Stipendien, ohne diese bevorzugt den Nachkommen der Bäcker zur Verfügung zu stellen. ${ }^{45}$

Auffallend für die Johnsche Stiftung von 1644 war nun, dass ein Nicht-Handwerker (ein herzoglicher Sekretär) ein Legat exklusiv für eine einzelne Handwerksinnung aufsetzte. Im Kurfürstentum Sachsen und seinen Sekundogenituren fand sich keine vergleichbare Stipendienstiftung. Diese Form der Festlegung der Empfängerschaft kann somit als besonders markant gelten. Nur die Söhne von Schneidern sollten die Jahreszinsen des legierten Kapitals in Höhe von 30 Reichstalern erhalten. Genauere Regelungen zum Verwaltungsmodus der Gelder fehlten. Das hieß effektiv, ein weiteres Spezifikum, dass die Schneiderinnung zu Leipzig jene fünfhundert Reichstaler Stiftungskapital selbst anlegen konnte und das allei-

Politik, Schule und Bildungsvermittlung in der vormodernen Stadtgesellschaft. Das Kurfürstentum und Königreich Sachsen 1600-1815 (Contubernium 78), Stuttgart 2012, besonders S. 162 f., 169.

42 Leipziger Beispiele: Geffcken, Stiftungsbuch (wie Anm. 13), S. 50 f., Nr. 70; S. 99, Nr. 177; S. 114, Nr. 205; S. 325, Nr. 507b u. ö.; LANGE, Kürschner-Innung (wie Anm. 40), S. $12 \mathrm{f}$.

43 Einzelne Beispiele außerhalb Leipzigs finden sich bei: Johann Christian Hasche (Hg.), Magazin der Sächsischen Geschichte 4 (1787), S. 353; Huemer, Kiel (wie Anm. 36), S. 46; Riegel, Kitzingen (wie Anm. 5), S. 111. Für Nürnberg konnte Ebneth je nach Zählweise immerhin 14 bis 15 Handwerks-Stipendienstiftungen nachweisen. Die relativ hohe Zahl ergibt sich vor allem aus der engen Verwobenheit einiger (ratsfähiger) Handwerke mit dem Stadtregiment. Eвneth, Nürnberg (wie Anm. 1), besonders S. 151, 166-169, 176.

44 Als Beleg führt Ebneth zwei Stiftungen aus dem Nürnberger Goldschmiedehandwerk an. Ebneth, Nürnberg (wie Anm. 1), S. 176. Vgl. Theodor Hampe, Nürnberger Ratsverlässe über Kunst und Künstler im Zeitalter der Spätgotik und Renaissance (1449) 1474-1618 (1633), Bd. II: 1571-1618 (1633), Wien/Leipzig 1904, S. 104, Nr. 620.

45 Geffcken, Stiftungsbuch (wie Anm. 13), S. 145, Nr. 256; RatAJszczak, Bildungspolitik (wie Anm. 1), S. 124 f. Eine dritte Stiftung umfasste zwar nicht die Vergabe von finanziellen Mitteln an Studierende, wohl aber die Einrichtung von Freitischen. Sie war im 17. Jahrhundert durch Wenzel Buhle, den Obermeister des Leipziger Kürschnerhandwerks errichtet worden und sollte Studenten und Magistern aus Schlesien zugutekommen. StadtA Leipzig, Handwerkerinnungen (im Folgenden: Inn) Kürschner B 3, fol. 176v. Diese Teilstiftung ist ebenfalls weder im Stiftungsbuch noch in anderen einschlägigen Stiftungsverzeichnissen erwähnt. Vgl. Baumgart, Stipendien (wie Anm. 14); Geffcken, Stiftungsbuch (wie Anm. 13), S. 174 f., Nr. 319; Meltzer, Verzeichniß (wie Anm. 14). 
nige Präsentations- und Vergaberecht des Stipendiums besaß. In der Praxis kamen damit ausschließlich die Söhne von L e i p z i g e r Schneidermeistern in den Stipendiengenuss.

Die Erklärung für die ungewöhnliche Stiftungskonstellation liegt in Michael Johns sozialer Herkunft begründet. Mithilfe der Kirchenbücher und der von der städtischen Leichenschreiberei geführten Leichenbücher kann nachgewiesen werden, dass Michaels Vater Christoph John (sen.) aus Sade (vermutlich Sayda) bei Freiberg in Sachsen stammte und in der Messestadt sowohl das Bürgerrecht als auch das Meisterrecht als Schneider erwarb. Er heiratete 1602 Katharina, die eheliche Tochter des Leipziger Bürgers und Schneidermeisters Nickel Lauterbach. ${ }^{46}$ Die Familie unterhielt somit enge Beziehungen zur entsprechenden Handwerksinnung. Unter den drei Paten Michael Johns befand sich folgerichtig auch ein Schneider, ein Meister namens Elias Schaup, was auf freundschaftliche Verbindungen unter den Berufskollegen schließen lässt. Der rasante Aufstieg des Stifters vom einfachen Schneidersohn zum herzoglichen Sekretär ging in den unruhigen Kriegszeiten mit der Anhäufung eines durchaus beachtlichen Vermögens von mehreren Tausend Talern einher. ${ }^{47}$ Sicherlich auch aus Dankbarkeit gegenüber der glücklichen Fügung des eigenen Werdegangs und um anderen Schneidersöhnen eine bessere berufliche Perspektive zu eröffnen, erfolgte die Stipendienstiftung.

Dass der gewählte Verwaltungsmodus durch die Handwerksinnung dazu beitrug, die Stiftung fast gänzlich aus der Überlieferung verschwinden zu lassen, wurde bereits erwähnt. Dem Stifter schien die Überantwortung des Kapitals an die jahrhundertealte Innung sicher genug, um seinen Stifterwillen langfristig zu erfüllen. Die Handwerksinnung als halböffentliche Korporation gewährleistete, anders als eine Privatperson oder eine Familie, größere Dauerhaftigkeit der Stiftung. Noch dazu handelte es sich bei dem Leipziger Schneiderhandwerk um ein Massengewerbe, indem die Innung vor Kriegsausbruch knapp 100 und bei Aufsetzung des Testaments noch immer mindestens 56 Schneidermeister als Mitglieder aufwies, womit sie zu den größten Handwerksorganisationen der Stadt zählte. ${ }^{48}$

46 KA Leipzig, Traubuch St. Nicolai 1597-1608, S. 169; StadtA Leipzig, Ratsleichenbuch Nr. 10 (1633-1638), fol. 102v. Vermutlich ein Bruder des Vaters namens Christoph kam ebenfalls nach Leipzig, heiratete eine Vikarstochter und versuchte sich als Schreiber. KA Leipzig, Traubuch St. Thomas 1609-1646, fol. 156v.

47 Zum Vergleich der Vermögensgrößen siehe Wolfgang Feige, Die Sozialstruktur der spätmittelalterlichen deutschen Stadt im Spiegel der historischen Statistik - mit besonderer Berücksichtigung der niederen Schichten der Bevölkerung und mit einem Exkurs in das Leipzig des 16. Jahrhunderts, Diss. phil. Leipzig 1965, Bd. 2, S. 238-289.

StadtA Leipzig, Tit. LXIV (F) 151, Vol. I, fol. 28v; Tit. LXIV (F) 266, fol. 58v-59r; Tit. LXIV (F) 269, fol. 25r; Herbert Germar, Das Schneiderhandwerk in Leipzig bis zum Ausgange des 17. Jahrhunderts, Diss. phil. Weida in Thüringen 1918, S. 47 f. Vgl. die retrospektive Aussage in: StadtA Leipzig, Inn Schneider B 2, fol. 202r-202v. 


\section{Die Überlieferungssituation}

Die Informationslage zur Johnschen Stipendienstiftung ist dürftig. Eigenständige Stiftungsaufzeichnungen in Form von Stiftungsbüchern, Antrags- oder Empfängerlisten wurden nicht geführt. Daher mussten die Informationen punktuell aus den unterschiedlichsten archivalischen Quellen (Ratsbüchern, Zunftakten, Gerichtsakten, Kirchenbüchern, Matrikelbüchern etc.) gewonnen werden.

Ein besonderes methodisches Problem bildet die unvollständige Überlieferung der Schneiderinnung selbst, die zur Thematik doch den wichtigsten Teil der Informationsgewinnung beiträgt. Die Handwerksbücher, -protokolle und -rechnungen gelangten in Leipzig - wie in vielen anderen Städten - nur partiell, häufig durch Zufälle wie Schenkungen oder antiquarische Ankäufe, in staatliche, kommunale oder private Archiveinrichtungen. Dennoch konnten vor allem durch die Handwerksbücher die Namen von Dutzenden Stipendienbewerbern nachgewiesen werden. In der Regel gaben die Einträge in den Büchern nur den Familiennamen des Bewerbers preis. Selbst dürftige zusätzliche Informationen wie der Vorname des Bewerbers oder derjenige seines Vaters fehlten oft und konnten erst durch Hinzuziehung weiterer Quellenbestände ergänzt werden. Insbesondere durch Zuhilfenahme der Kirchenbücher und der Universitätsmatrikel konnte in der Mehrzahl der Fälle im Prinzip zweifelsfrei die Identität des Stipendienbewerbers und späteren Studierenden nachgewiesen werden. Bei den wenigen unsicheren Zuordnungen lagen entweder mehrere namensgleiche Einträge in den Quellen (z. B. den Taufbüchern oder Matrikeln) vor oder die Personen waren nicht in Leipzig geboren bzw. verstarben andernorts, sodass entsprechende Einträge in den Leipziger Kirchen- und Ratsleichenbüchern fehlten.

Die Informationen zum Stipendium und zu den Stipendienbewerbern verteilten sich über die Zeitläufe sehr unterschiedlich. Aus dem 17. Jahrhundert sind sieht man vom Codicill und von dessen Abschriften ab - keinerlei Hinweise vorhanden. Nur bei Herbert Germar ist zu lesen, dass kurze Zeit nach Michael Johns Tod, die Stiftung anscheinend gangbar wurde. Schon am 3. Mai 1645 soll ein Student, ein gewisser Christoph Knauer, als erster um das Stipendium bei der Schneiderinnung nachgesucht haben. Dessen schriftliches Gesuch habe sich noch Anfang des 20. Jahrhunderts im Besitz der Innung befunden. ${ }^{49}$ Germars Ausführungen erscheinen nach Überprüfung der Leipziger Universitätsmatrikel und der Taufbücher glaubhaft. Ein aus Leipzig stammender Christoph Knauer wurde 1625 in die Matrikelliste als non iuravit eingetragen und leistete einige Jahre später den Eid gegenüber der Hochschule..$^{50}$ Vermutlich handelte es sich dabei um den am 22. Dezember 1612 getauften Sohn des Schneidermeisters Christoff Knauer und seiner Ehefrau Maria. ${ }^{51}$

49 Germar, Schneiderhandwerk (wie Anm. 48), S. 91, Anm. 3.

50 ERLER, Matrikel I (wie Anm. 7), S. 229.

51 KA Leipzig, Taufbuch St. Nicolai 1609-1615, S. 108. Vgl. KA Leipzig, Taufbuch St. Nicolai 1601-1606, S. 329. 
Für das frühe 18. Jahrhundert finden sich im Leipziger Stadtarchiv erstmals Handwerks- und Protokollbücher der mit der Verwaltung der Stipendienstiftung betrauten Schneiderinnung, weshalb sich die Informationsdichte für unsere Thematik deutlich erhöht. ${ }^{2}$ Dennoch enthalten die Bücher nur sporadisch Einträge zu Bewerbungen und Vergabemodalitäten des Stipendiums. Teilweise werden Konflikte (z. B. bei der Stipendienzuteilung) sichtbar. ${ }^{53}$

Obwohl die allgemeinen Aufzeichnungen im letzten überlieferten Handwerksbuch der Leipziger Schneider bis 1847 reichen, versiegen die unregelmäßigen Hinweise auf das Johnsche Stipendium am Ende des 18. Jahrhunderts. Die Stiftung soll zwar mindestens noch bis zum Ende des Ersten Weltkrieges bestanden haben, ${ }^{54}$ jedoch sind nach 1847 keine Handwerks-, Protokoll- oder Rechnungsbücher der Schneiderinnung mehr überliefert. ${ }^{55}$ Wie andere Studien belegen, nahm die Bedeutung der Stipendien für die Studierendenschaft seit dem Ende des 19. Jahrhunderts ohnehin aufgrund der enorm gestiegenen Studierendenzahlen drastisch ab. ${ }^{56} \mathrm{Ob}$ die Stiftung die Hyperinflation der 1920er-Jahre überlebte, ist ungewiss. ${ }^{57}$ Ihre Spuren verlieren sich - wie bei vielen anderen (Stipendien-)Stiftungen - endgültig im 20. Jahrhundert.

\section{Stiftungswirklichkeiten (1): Bewerbung und Empfangsbedingungen}

Um eine Studienförderung durch ein Stipendium zu erhalten, mussten die potenziellen Empfänger erst einmal von dieser Möglichkeit überhaupt wissen. Dies stellte im Falle der Johnschen Stipendienstiftung - anders als bei vielen städtisch oder universitär vergebenen Unterstützungen - keine echte Hürde dar, da die Väter der Studierwilligen als Handwerksmeister gemeinhin die Zunftversammlungen besuchten, auf denen über die Stipendienvergabe beraten wurde. ${ }^{58}$

52 Rechnungsbücher der Leipziger Schneiderinnung fehlen dagegen völlig.

53 Ergänzend konnte auf einzelne Akten der sogenannten II. Sektion zugegriffen werden, die wiedergeben, wie dem Rat einige Streitfälle bezüglich der Stipendienvergabe zur Entscheidung vorgelegt wurden.

54 Germar, Schneiderhandwerk (wie Anm. 48), S. 91, Anm. 3.

55 Es wurden die Bestände folgender Archiveinrichtungen diesbezüglich überprüft: Stadtarchiv Leipzig, Sächsisches Staatsarchiv - Staatsarchiv Leipzig, Sächsisches Wirtschaftsarchiv e. V., Archiv der Handwerkskammer zu Leipzig.

56 Adam, Stipendienstiftungen (wie Anm. 9), S. 135, 137, 149 f.; Auge, Zum Nutzen (wie Anm. 3), S. 161.

57 Zur Vernichtung großer Stiftungskapitalien zwischen dem Ersten Weltkrieg und der Hyperinflation 1923 sowie zur Aufhebung privater Stiftungen in der Sowjetischen Besatzungszone nach dem zweiten Weltkrieg siehe ADAM, Stipendienstiftungen (wie Anm. 9), S. 93-97; Auge, Zum Nutzen (wie Anm. 3), S. 159-162; Eвneth, Stipendium (wie Anm. 1), S. 520.

$58 \mathrm{Zu}$ den Bewerbungs- und Vergabemodalitäten siehe zum Vergleich: EBNETH, Nürnberg (wie Anm. 1), S. 217-225. Der Begriff ,Stiftungswirklichkeiten' wurde unter anderem verwendet von: Auge, Zum Nutzen (wie Anm. 3); Michael Borgolte (Hg.), Stiftungen und Stiftungswirklichkeiten. Vom Mittelalter bis zur Gegenwart (Stiftungsgeschichten 1), Berlin 2000. 
Die Stipendienkandidaten mussten sich für die Vergabe bewerben, wobei den Vätern schon allein aufgrund des zum Teil noch recht jugendlichen Alters der Kandidaten eine entscheidende Rolle zukam. Die Bewerber (bzw. deren Väter) verfassten Bittschreiben oder sprachen auf einer Versammlung des Handwerks persönlich vor.

$\mathrm{Zu}$ welchem Zeitpunkt die Bewerbung eines Kandidaten für gewöhnlich erstmals erfolgte, ist nicht sicher zu sagen. In den Akten und Handwerksbüchern finden sich, wenn überhaupt, nur zufällig Anmerkungen zu den schriftlichen und mündlichen Bewerbungen. Vielfach ist der genaue Zeitpunkt unbekannt. In allen Fällen, in denen man mehr oder weniger sichere Kenntnis von der ersten Vorsprache gewinnen kann, waren die Schneidersöhne bereits vor ihrer ersten Bewerbung in die Leipziger Universitätsmatrikel eingetragen worden. Auch ein Versprechen, den universitären Verpflichtungen nachzukommen, hatten alle schon abgelegt. Nur Meister Johann Georg Wilhelm Mieser reichte mehr als ein halbes Jahr vor Ableistung des universitären Gehorsamsversprechens seines Sohnes Gottlieb Wilhelm, der zumindest als iniurati eingeschrieben war, ein Bewerbungsschreiben an die Innung ein.

Bei schriftlichen Bewerbungen wurden die Briefe und Supplikationen vor dem Handwerk verlesen. Bei mündlicher Vorsprache hörten sich die Meister das Anliegen als einen von mehreren Tagesordnungspunkten auf ihrer Zunftversammlung an. Über die Stipendienvergabe wurde, wie bei innerzünftigen Diskussions- und Streitthemen üblich, mehrheitlich bzw. nach Mehrheit der Tische abgestimmt. ${ }^{59}$ Zur Urteilsfindung berücksichtigten die Meister verschiedene Punkte. Wem kam also die Johnsche Stipendienstiftung zugute? Welche Bedingungen mussten für den Empfang des Stipendiums erfüllt werden?

Das stipendio sollte einem Schneidersohn, das bedeutete in der Praxis dem Sohn eines Mitglieds der Leipziger Schneiderzunft, zu beßer Fortsetzung seines Studirens über drei Jahre gereicht werden. Testamentarisch war ein Studium an der Leipziger Universität keine ausdrückliche Voraussetzung für den Empfang des Stipendiums. De facto handelte es sich wohl jedoch um eine solche Festlegung, ${ }^{60}$ studierten doch alle nachweisbaren Stipendienempfänger in Leipzig. Eventuell legte die das Vergaberecht innehabende Schneiderinnung Wert darauf. Von Rele-

59 Die innerzünftige Organisation der Leipziger Schneider sah eine Gliederung der Mitgliederschaft auf 13 Tische vor. Die Meister saßen abhängig vom Alter des Erwerbs ihres Meisterrechts an einem der Tische. Diejenigen, welche am längsten Innungsmitglieder waren, beispielsweise am ersten oder ältesten Tisch.

60 Vgl. Germar, Schneiderhandwerk (wie Anm. 48), S. 91. Die offen gestaltete Regelung trifft sich mit der Feststellung bei Bahlke, wonach private Stiftungen prinzipiell oft nicht an den Landesgrenzen haltmachten. Joachim BaHLke, Bergesche Stipendien. Zielsetzung und Indienstnahme einer frühneuzeitlichen Studienstiftung im konfessionellen Zeitalter, in: Joachim Bahlke/Thomas Winkelbauer (Hg.), Schulstiftungen und Studienfinanzierung. Bildungsmäzenatentum in den böhmischen, österreichischen und ungarischen Ländern, 1500-1800 (Veröffentlichungen des Instituts für Österreichische Geschichtsforschung 58), Wien/München 2011, S. 129-151, hier S. 150. 
vanz war sicherlich zudem das preiswertere Leben und Studium der Söhne von Leipziger Handwerkermeistern in ihrer Heimatstadt. ${ }^{61}$ Weitere Vergabekriterien nannte das Johnsche Testament nicht.

In der Forschungsliteratur werden meist noch ,Würdigkeit' und ,Bedürftigkeit ${ }^{`}$ als übliche Mindestanforderungen für den Empfang eines Stipendiums genannt. ${ }^{62}$ Inwiefern dies auf das Schneider-Stipendium zutraf, ist zumindest teilweise fraglich. Der Meistersohn Johann Christoph Wolckau sollte, weil er nicht persönlich vorsprach, sondern auswärts weilte, sich Legitimiren und ein gut Adestat bringen, wie er sich verhalten hat. Atteste wie dieses wurden auf ihre Richtigkeit überprüft. ${ }^{63}$ Anscheinend hatten die Bewerber Nachweise über die angelaufenen universitären Studien zu erbringen, wobei die tatsächlichen Studienleistungen wohl nicht kontrolliert wurden, wenngleich die Innung mangelnden Studienerfolg bei Konflikten vor Gericht als Argument anführte. ${ }^{64}$ Gleich drei Leistungspatente legte in einem Streitfall Carl Heinrich Jahn vor. ${ }^{65}$ Auch im Handwerksbuch findet sich im Jahr 1779 ein einschlägiger Eintrag: Christlieb Friedrich Kirchner, ein biesiger hinterlaßner Meisters Sohn, ist vor einigen Jabren als Gesell eingeschrieben, denselben ist eingefallen die studiu zu Frequendiren. [Er] bringt ein Zeugniß von $H$ [errn] Hof R [at] Beel, das er alda schon 1/2 Jahr Collegia geböret, [und] verlangt das gewöhnl[iche] Stibendium. ${ }^{66}$ Kirchner blieb mit diesem ersten Gesuch erfolglos. Als er drei Jahre später nochmals vorsprach, wurde er erneut abgewiesen, $d a$ er ein Sprachmeister in Franckreich hette machen wollen, [auch] die acattemi und Collegi Quittirt [habe], so könde er nichts davon genießen, indem er sich selbst darum gebracht hette. ${ }^{67}$ In einem anderen Fall versuchte ein Kandidat einen Mitbewerber zu diskreditieren, indem er behauptete, der andere wäre längst kein Student mehr. ${ }^{68}$

Manche Anwärter versuchten, die ,Ehrbarkeit' der eigenen Familie herauszustellen und diejenige anderer anzuzweifeln. Dies konnte geschehen, indem die Väter anderer Bewerber beschuldigt wurden, gegen die Zunftregeln zu verstoßen, wenn jene beispielsweise über das statutarisch festgelegte Maß hinaus Arbeits-

61 An die kostenlose Unterkunft im Elternhaus kamen selbstverständlich selbst die günstigen Studentenunterkünfte im Paulinum nicht heran. BRUCHMÜLlER, Typus (wie Anm. 8), S. 336; Ders., Der Leipziger Student (wie Anm. 8), S. 90. Vgl. PoHL, Lebensführung (wie Anm. 12), S. 221.

62 Adam, Stipendienstiftungen (wie Anm. 9), S. 122-129; Ratajszczak, Bildungspolitik (wie Anm. 1), S. 108.

63 StadtA Leipzig, Inn Schneider B 2, fol. 180r, 196r.

64 StadtA Leipzig, II. Sektion I (F) 101, fol. 31r.

65 Ebd., fol. 12r, 13r, 14r.

66 StadtA Leipzig, Inn Schneider B 11, fol. 93v. Karl Andreas Bel (1717-1782) war seit 1756 bis zu seinem Tode ordentlicher Professor für Poesie und mehrfacher Rektor der Universität Leipzig. Internet-Ressource: http://www.uni-leipzig.de/unigeschichte/ professorenkatalog/leipzig/Bel_1349/[Zugriff 8. April 2015].

67 StadtA Leipzig, Inn Schneider B 11, fol. 138v. Später entschied sich die Innung doch noch um. Ebd., fol. 177r, 198v.

68 StadtA Leipzig, II. Sektion O (F) 92, fol. 12v. 
kräfte beschäftigten. Berufsbezogene Vorwürfe wie dieser tangierten zum einen die ,Ehrbarkeit', zum anderen aber die ,Bedürftigkeit', denn die Fähigkeit, mehr als üblich Gesellen und Lehrlinge zu unterhalten, deutete auf eine eher günstige ökonomische Situation als Meister hin.

$\mathrm{Ob}$ es während des Stipendienbezugs weitere Überprüfungen der Kandidaten gab und die Innung somit disziplinierend auf das Verhalten der Stipendiaten einwirken konnte, bleibt ebenso Spekulation wie die Existenz eines formalisierten Prüfungswesens, welches sich bei den städtischen und universitären Behörden für die Stipendienbewerber durchaus herausbildete. ${ }^{69}$

In den Bewerbungen gegenüber der Zunft (oder im Klagefall gegenüber dem Stadtrat) betonten die Kandidaten bisweilen ihre erbärmliche finanzielle Lage oder die ihrer Familie. Der Schneidersohn Carl Heinrich Jahn bat um die Unterstützung, weil, wie er über sich sagte, ich ein blutarmer Mensch bin, und bloß von informiren mich kümmerlich erbalten, und von meinen armen Eltern obne dem keine Assistence mir versprechen kan. ${ }^{70}$ Erfolg war dem Ansuchen Christian Traugott Creutzers um eine zeitliche Verlängerung der Förderung beschieden. Da er noch keine Versorgung hatte, sollte Creutzer das Stipendium nach Handwerksbeschluss ein weiteres Jahr erhalten. ${ }^{71}$

Sowohl, Würdigkeit‘ als auch ,Bedürftigkeit‘ müssen als relative Bedingungen angesehen werden. Zur genaueren Überprüfung des Kriteriums ,Bedürftigkeit‘ wäre sicherlich eine Analyse der einschlägigen Leipziger Steuerlisten hilfreich, doch kann eine solche Untersuchung an dieser Stelle nicht erfolgen. Auch die unzureichende Informationsbasis zur tatsächlichen Stipendienvergabe verwehrt eine abschließende Antwort auf diese Frage. In einem Einwohnerverzeichnis aus dem Jahre 1771 finden sich zumindest einige der Schneidermeister wieder. Sie sind dort ausnahmslos als Mieter und nicht als Hausbesitzer aufgeführt. ${ }^{72}$

Die These einer deutlichen Bevorzugung von Söhnen der zünftigen Funktionselite konnte nicht verifiziert werden. Unter den nachgewiesenen Stipendienempfängern befinden sich nur ein Sohn eines Vormeisters und späteren Obermeisters (Johann Friedrich August Hey) und ein Sohn eines späteren Vormeisters (Christoph Friedrich Trück). Bei zwei weiteren Bewerbern, handelt es sich vermutlich um den Sohn eines Obermeisters (Johann Gottfried Grünthal) und den Sohn eines Vormeisters (Johann Gottlieb Mieser), doch konnte bei ihnen der Stipendienempfang nicht belegt werden. ${ }^{73}$ Wiewohl also nicht nur Söhne der zünf-

69 Ebneth, Stipendium (wie Anm. 1), S. 527, 530; Löfrler, Privatstipendien (wie Anm. 4), S. 360 f.

70 StadtA Leipzig, II. Sektion I (F) 101, fol. 1v.

71 StadtA Leipzig, Inn Schneider B 11, fol. 52v.

72 StadtA Leipzig, Tit. XLII D (F) 153.

73 Für die Quellenbelege siehe die Tabellen 1 und 2 im Anhang. Die drei Obermeister des Leipziger Schneiderhandwerks (davon ein regierender, amtsführender Obermeister) standen den Versammlungen der Zunftgemeinschaft vor, vertraten die Organisation gegenüber dem Landesherrn, dem Stadtrat und anderen Institutionen und straften nach den Artikeln der Zunftordnung. Ihnen hatten alle anderen Innungsmitglieder Gehorsam 
tigen Amtselite das Stipendium erhielten, legte man doch bei deren Bewerbung womöglich eine etwas großzügigere Vergabepraxis an den Tag. Der erst 18-jährige Johann Friedrich Regel junior erhielt das Stipendium nur anderthalb Jahre nach seiner Immatrikulation und damit deutlich früher als viele andere Bewerber. Sein Vater war einige Monate zuvor verstorben, sein Vormund war der Schneider und Handwerksvormeister Christian Wilhelm Weniger. Das Stipendium wurde zudem zwischen Regel junior und einem weiteren Bewerber, der sich vor dem jungen Regel beworben hatte, geteilt und auf vier Jahre gereicht. ${ }^{74}$ Die Analyse der Taufpaten der Stipendienempfänger gestattet ebenfalls nicht, von einer klaren Tendenz zur vorrangigen Unterstützung jener Schneidersöhne zu sprechen, deren Väter ein höheres Maß an Sozialprestige oder ein größeres Vermögen vorweisen konnten. ${ }^{75}$

Als mögliches Hemmnis für die Aufnahme eines Studiums durch ärmere Personen kam eine geringe Stipendienhöhe in Betracht. Bei Auflegung der Johnschen Stiftung dürften 30 Reichstaler durchaus ein für Leipziger Verhältnisse üblicher, wenngleich wohl recht knapp bemessener Stipendienbetrag gewesen sein. ${ }^{76} \mathrm{Je}$ länger allerdings die in ihrer Größenordnung unveränderte Stiftung bestand, desto weniger konnte sie die tatsächlichen Lebenshaltungskosten eines Leipziger Studenten decken. Hinzu kam, dass anscheinend im 18. Jahrhundert statt 30 nur 25 Reichstaler jährlich ausgereicht wurden, ohne dass ein Grund benannt wurde. Dieser Betrag diente nur als ein Zubrot für die Studierenden. ${ }^{77}$ Spätestens nach

zu leisten. Die drei Vier- oder Vormeister des Handwerks (davon ein regierender Vormeister) führten die Rechnung der Leichenkasse und unterstützten die Obermeister bei ihren Aufgaben. Ferner wurden drei Beisitzer bei der Meister- und zwei Beisitzer bei der Gesellenlade durch Mehrheitswahl bestimmt. Weitere Funktionsämter wie diejenigen der Störrottenmeister (für das ,Aufheben' von Störern) und der Einwerbemeister (für die Einladung zu den Quartalsversammlungen) besaßen kein hohes Prestige innerhalb des Handwerks.

74 KA Leipzig, Taufbuch Nicolai 1764-1771, S. 687, Nr. 456; StadtA Leipzig, Inn Schneider B 11, fol. 210v; Georg Erler (Hg.), Die jüngere Matrikel der Universität Leipzig 1559-1809. Als Personen- und Ortsregister bearbeitet und durch Nachträge aus den Promotionsakten ergänzt, Bd. III: Die Immatrikulationen vom Wintersemester 1709 bis zum Sommersemester 1809, Leipzig 1909, S. 318.

75 Von den gut 70 Paten, zu denen Angaben zum Berufsstand vorliegen, kamen etwa $40 \%$ aus dem Handwerk. Etwas über 20 \% der Paten waren Handelsleute, Kramer oder deren Familienangehörige. Vereinzelt fanden sich beispielsweise Ratsherren, städtische Angestellte, Geistliche und Mediziner.

76 Die kursächsischen Landesstipendien in Leipzig und der sächsischen Schwesteruniversität Wittenberg beliefen sich allerdings bereits Anfang des 17. Jahrhunderts alle auf 40 oder 50 Gulden jährlich. Dagegen betrug die allgemeine durchschnittliche Stipendienhöhe in Leipzig im 16. Jahrhundert etwa 20 bis 25 Gulden und diejenige der sächsischen Landesstipendien 30 bis 40 Gulden (Ausnahme: vier höhere Stipendien über 90 Gulden). Gössner, Wittenberg (wie Anm. 4), S. 118 f.; Ratajszczak, Bildungspolitik (wie Anm. 1), S. 137 f. Zu den Nürnberger und Wittenberger Verhältnissen vgl. EBNETH, Nürnberg (wie Anm. 1), S. 213, 216; RatajszczaK, Bildungspolitik (wie Anm. 1), S. 140.

77 Während der Original-Codicill und die diversen Abschriften von 30 Reichstalern sprechen, beziehen sich zahlreiche Aufzeichnungen, in denen es um die praktische Auszahlung des Stipendiums geht, auf eine jährliche Jahresunterstützung von 25 Reichstalern. Eventuell spielte das übliche Zinsniveau bei der geringeren Auszahlung 
einem erheblichen Anstieg der Lebenshaltungskosten im weiteren Verlauf des 18. Jahrhunderts waren die Stipendienempfänger auf zusätzliche Einkommensquellen angewiesen. Eine zeitgenössische Berechnung kam Ende des 18. Jahrhunderts für Leipzig auf einen jährlichen Mindestlebensstandard, für den etwa 120 Reichstaler nötig gewesen seien. ${ }^{78}$ Auf die vorhandenen und zugleich lebensnotwendigen zusätzlichen Erwerbsmöglichkeiten für Studierende (mit und ohne Stipendium) sei lediglich allgemein hingewiesen. ${ }^{79}$ Der gleichzeitige Bezug mehrerer Stipendien war grundsätzlich ebenfalls eine Möglichkeit. ${ }^{80}$

Bedeutsamer als das Kriterium ,Bedürftigkeit‘ war neben den weiteren Lebensumständen (ein Bewerber wurde abgewiesen, weil er schon etl[iche] $50 \mathrm{Jahr}$ alt und verheiratet war ${ }^{81}$ ) der Nachweis der sozialen Herkunft als Schneidersohn. So wurde Gottfried Lange nicht als Sohn eines Leipziger Schneidermeisters anerkannt, weshalb er neben anderen Gründen (verheiratet, in Diensten stehend) das Stipendium nicht erhielt, vermutlich aber dennoch studierte. ${ }^{82}$ Ein anderer Fall beschäftigte die Innung, den Stadtrat und die Gerichte vor Ort über mehrere Jahre. ${ }^{83}$ Carl Heinrich Jahn wandte sich im März 1718 hilfesuchend an den Leipziger Magistrat, die Schneiderinnung verweigere ihm das Stipendium. Die Innung führte als ein Argument an, dass der Vater zur Zeit von Carl Heinrichs Navitaet noch kein Meister gewesen sei und den Sohn nicht mit der üblichen Gebühr ins Handwerk eingekauft habe. Auch Jahns lange Studienzeit von über neun Jahren wurde von der Zunft kritisiert, vermutlich sei Jahn gar kein Student mehr. Dessen ungeachtet beschloss der Stadtrat, sich an einem juristischen Gutachten zu orientieren, nach welchem Söhne, die von ,Nicht-Meistern' gezeugt wurden, als Meistersöhne anzuerkennen seien, sobald der Vater von der Innung zu einem ordent-

eine Rolle. Vgl. StadtA Leipzig, II. Sektion I (F) 101, fol. 1r, 20r; II. Sektion O (F) 92, fol. 19r; Inn Schneider C 14, fol. 23r; Richterstube, Testamente Rep. V, Paket 71, Nr. 2, fol. 1v; Stift. VIII B Nr. 1b, fol. 26r.

78 Friedrich GotTlob LeONHARdi, Allgemeine theoretisch-praktische Stadt- und Landwirthschaftskunde, Bd. 1, 1. Stück, Leipzig 1789, S. 61. Löffler berechnet den finanziellen Aufwand selbst mit etwas über 100 Talern. Löffler, Privatstipendien (wie Anm. 4), S. 367; Katrin Löffler (Hg.), Als Studiosus in Pleiß-Athen. Autobiographische Erinnerungen von Leipziger Studenten des 18. Jahrhunderts, Leipzig 2009, S. 9. Weitere Kostenbeispiele in: DörIng, Anfänge (wie Anm. 11), S. 603-605.

79 Bruchmüller, Typus (wie Anm. 8), S. 337; Leonhardi, Stadt- und Landwirthschaftskunde (wie Anm. 78), S. 61 f.; PoHL, Lebensführung (wie Anm. 12), insbesondere S. 218-220, 227.

80 Döring, Anfänge (wie Anm. 11), S. 611; Löffler, Privatstipendien (wie Anm. 4), S. 369 .

81 StadtA Leipzig, Inn Schneider B 11, fol. 51r.

82 StadtA Leipzig, Inn Schneider B 2, fol. 153r. Der Name Gottfried Lange kann nicht sicher einem Leipziger Matrikeleintrag zugeordnet werden, da mehrere Treffer existieren. Am wahrscheinlichsten trifft der Immatrikulationseintrag von Christian Gottfried Lange vom Sommersemester 1714 auf den Stipendienbewerber zu. ERLER, Matrikel III (wie Anm. 74), S. 227.

83 Zum folgenden Fall siehe StadtA Leipzig, II. Sektion I (F) 101; Inn Schneider B 2, fol. $150 v, 180 \mathrm{r}, 202 \mathrm{r}-202 \mathrm{v}, 213 \mathrm{v}, 219 \mathrm{v}, 247 \mathrm{r}$. 
lichen Handwerksmeister gesprochen worden war. Das Gutachten zog konsequenterweise eine Ratsresolution nach sich, Jahn das Stipendium zuzusprechen. Unter vielerlei Einwänden widersetzte sich die Zunft und verschleppte immer wieder die Auszahlung des Stipendiums. Im Juni 1719 konnte das Urteil des zusätzlich angerufenen Schöppengerichts verkündet werden. Es fiel zugunsten von Jahn aus. Als letzte Möglichkeit drohte die Innung nun damit, an die kursächsische Landesregierung zu schreiben. Aus Sicht der Innung handelte man im Prinzip aus Notwehr. Das ,ehrbare' Handwerk hätte einen zu großen Praejudiz, wenn der Stifterwillen so gedeutet würde, dass auch Söhne von Pfuschern das Stipendium erhalten könnten. In diesem Sinne war der Bewerber in den Augen der Zunft nicht stipendienwürdig. Man schreckte dann aber vor den hohen Kosten zurück und beugte sich scheinbar dem Gerichtsurteil. Als Carl Heinrich Jahn auf der nächsten Innungsversammlung auftrat, erklärte man ihm, wenn er das Stipendium erhalten wolle, so solte er sich erstlich mit dem handwercke abfinden. Wieder zog Jahn vor den Stadtrat. Die Ratsherren ermahnten die Schneider bei zehn Talern Strafe, dem Urteil vom Juni nachzufolgen und das Stipendium an Jahn gehen zu lassen. Schließlich sagte die Zunft zu, dass Jahn das Stipendium ab Ostern des Folgejahres erhalten solle. Dass die Zunft nicht klein beigab, sollte sich schon 1722 und also noch vor Ablauf der Bezugsdauer von drei Jahren zeigen. Jahn musste damals erneut um die Auszahlung seiner restlichen Stipendiengelder kämpfen.

\section{Stiftungswirklichkeiten (2): Der Stipendienempfang}

Selbst wenn die Zunft mit einem Bewerber zufrieden war und ihm den Empfang des Schneider-Stipendiums zusagte, verhinderten häufig andere Hürden einen baldigen Bezug. Leider wurde kein Quittungsbuch über die Auszahlung der Stipendiengelder geführt. Nur eine einzige Quittung, die vermutlich aus besonderem Grund angefertigt wurde, hat sich erhalten. ${ }^{84}$ In 18 Fällen kann definitiv nachgewiesen werden, dass es zu einer Zusage über die Auszahlung der Stipendienmittel kam. In diesen Fällen kann der ungefähre Zeitpunkt des erstmaligen Stipendienempfangs bestimmt werden. Obwohl im Johnschen Testament nichts Genaueres festgelegt worden war, verlangte die Innung anscheinend Adestate über den Status als Studierender. Auch aufgrund enormer Nachfrage und vielfach mehrerer Bewerber in einem Jahr, erhielten die Stipendiaten ihre Gelder erst Jahre nach Studienbeginn. Bei Zusage empfing ein Kandidat im günstigsten Fall das Stipendium anderthalb, im schlechtesten Fall elf Jahre nach dem jeweiligen Matrikeleintrag. Im Durchschnitt genoss ein Schneidersohn, dem der Empfang genehmigt wurde, sein Stipendium erstmals fünf bis sechs Jahre nach Studienbeginn. Manchmal wurden die 25 Reichstaler erst kurz nach der Verleihung des akademischen Grades magister artium gewährt. Das Stipendium konnte somit in den meisten Fällen 
nicht dazu dienen, die ersten Studienjahre zu finanzieren. So schlug sich ein Stipendienbewerber namens Johann Gottlieb Öser jahrelang als Famulus und Tanzlehrer durch. ${ }^{85}$ Eher kamen die Gelder Studenten im fortgeschrittenen Stadium ihrer Studien zugute. Dennoch erscheint es ausgeschlossen, dass das Stipendium regelmäßig nach Abschluss der Universitätsstudien gezahlt wurde. Vielmehr muss bei den meisten Empfängern von einem vieljährigen Universitätsbesuch ausgegangen werden.

Das Stipendium wurde gemäß der testamentarischen Vorgabe normalerweise für drei Jahre an einen aus Sicht der Schneiderinnung geeigneten Meistersohn vergeben. In einzelnen Fällen gab es Verlängerungen um ein halbes oder ein ganzes Jahr. Damit lag das Johnsche Stipendium bezogen auf die Vergabedauer von Privat- und Familienstipendien im (unteren) Durchschnitt. ${ }^{86}$

Waren mehrere geeignete Kandidaten vorhanden, entschied über die Vergabe des Stipendiums (neben den Beziehungs- und Patronagegeflechten innerhalb der Zunft) vor allem der Zeitfaktor. Man findet in den Quellen zwei Argumentationslinien, ohne völlig klären zu können, welche von beiden durchgängig bzw. zu welchen Zeiten angewandt wurde. Stipendienbewerber, die sich ungerecht durch die das Vergaberecht besitzende Schneiderinnung behandelt wähnten, rekurierten auf ihr im Vergleich zu ihren Konkurrenten früheres Immatrikulationsdatum bei der Universität. ${ }^{87}$ Dagegen argumentierte die Innung, dass der Zeitpunkt der Bewerbung auf der Zunftversammlung entscheidend sei und das Stipendium bei der Prüfung der Vergabekriterien versprochen wurde. ${ }^{88}$ Nach zünftiger Argumentation erhielt bei Erfüllung der übrigen Vorgaben im Regelfall also der den Vorzug, der sich zuerst um ein Stipendium beworben hatte. ${ }^{89}$ Diejenigen, welchen der Bezug grundsätzlich zugesagt worden war und die dennoch vorerst leer ausgingen, befanden sich in einer Art Warteposition und rückten entsprechend dem Stipendienzyklus nach jeweils drei Jahren nach. Aufgrund der zeitweise sehr hohen Nachfrage mussten die Bewerber mit langen Wartezeiten rechnen und zwar auch als man dazu überging, das Stipendium teilweise nur noch befristet für ein Jahr zu vergeben, teilweise es auf mehrere Kandidaten aufzuteilen. Letzteres geschah

85 StadtA Leipzig, II. Sektion O (F) 92, fol. 2r-2v.

86 Löffler, Privatstipendien (wie Anm. 4), S. 359; PoHl, Lebensführung (wie Anm. 12), S. 219; Ratajszczak, Bildungspolitik (wie Anm. 1), S. 107, 134, 138.

87 Diese Argumentation findet sich z. B. in der Eingabe des Kandidaten Johann Gottlieb Öser. Öser klagte vor dem Stadtrat 1724 auf Empfang des Stipendiums und meinte, die Observanz auf seiner Seite zu haben, nach welcher bey mebr als 50 Jabren her jederzeit der älteste Studiosus dieses Stipendium genoßen, und einer auf den andern zu wartten, zur Gedult, wie mir geschehen, verwiesen worden. Er selbst warte schon sieben Jahre darauf. Aufgrund der Beschwerde lenkte die Innung tatsächlich ein und vergab das Stipendium an Öser. StadtA Leipzig, II. Sektion O (F) 92, fol. 1v, 15v-16r, 21r; II. Sektion I (F) 101, fol. 1r.

88 StadtA Leipzig, Inn Schneider B 11, fol. 93v.

89 StadtA Leipzig, II. Sektion O (F) 92, fol. 16r-16v, 21 r. 
insbesondere am Ende des 18. Jahrhunderts. ${ }^{90}$ Aber schon um 1720 schien der Andrang groß zu sein. Allein 1724 stritten mindestens vier Anwärter um das Johnsche Stipendium. ${ }^{91}$

Nachdem es vermutlich immer wieder zu Diskussionen und Unstimmigkeiten über die korrekte Stipendienvergabe gekommen war, erfolgte spätestens 1789 eine Modifizierung der Vergaberegeln. Der Handwerksschreiber notierte ins Protokollbuch: Bey Votirung wegen des Stibentiums, wer es ins künftige bekomen solle, so wabren 11 Tüsche, deren Resolution wahr, das es der haben solte, deßen Vatter am lengsten Meister ist. ${ }^{92} \mathrm{Z}$ war kam es auch in der Folgezeit noch zur Teilung des Stipendiums, aber im Streitfall hatten nun die Söhne der dienstjüngeren Meister das Nachsehen. ${ }^{93}$

\section{Die Stipendienbewerber}

Durch Auswertung der städtischen und zünftigen Überlieferung konnten 30 Personen ermittelt werden, die sich um das Stipendium bewarben und/oder es erhielten. ${ }^{94}$ Über einen Abgleich dieser Quellen mit den Universitätsmatrikeln, den Kirchenbüchern und anderen Archivbeständen kann mit großer Wahrscheinlichkeit davon ausgegangen werden, dass alle diese 30 Schneidersöhne in Leipzig studierten und zwar unabhängig vom Bezug des Johnschen Stipendiums. Die Beispiele des Gottfried Lange oder des Carl Heinrich Jahn wurden erwähnt. Die Verweigerung des Stipendiums hielt keinen Studierwilligen vom Besuch der Alma mater ab. Dies lag oft schon an der erwähnten, vergleichsweise späten Vergabe des Stipendiums.

Eine breite Quellenrecherche ermöglichte die Sammlung weiterer biografischer Informationen zu fast allen der 30 Stipendienbewerbern. Vermutlich alle besuchten die Leipziger Artistenfakultät im Erststudium. Über den Abgleich von Kirchenbüchern und Matrikeln lässt sich das ungefähre Alter der Schneidersöhne bei Eintritt in die Universität ermitteln. Insbesondere die Matrikeleinträge bis zur ersten Hälfte des 18. Jahrhunderts unterscheiden dabei zwischen iniurati und iurati. Einige wurden schon vor Erreichen der Eidesfähigkeit in die Universitätsmatrikel eingeschrieben und legten erst später den akademischen Eid (bzw. seit

90 StadtA Leipzig, Inn Schneider B 11, fol. 177r, 279v, 290r. In einem Fall wurde das Stipendium für vier Jahre an zwei Meistersöhne zugleich vergeben. Ebd., fol. 210v.

91 StadtA Leipzig, II. Sektion O (F) 92, fol. 9r; Inn Schneider B 2, fol. 202r-202v.

92 StadtA Leipzig, Inn Schneider B 11, fol. 210v.

93 Ebd., fol. 259r.

94 Eine weitere Person, Johann Lorentz Stenden, wird im Handwerksbuch der Schneider zwar in Verbindung mit gewissen Stipendia erwähnt, doch scheint es sich hierbei nicht um das Johnsche Stipendium gehandelt zu haben. StadtA Leipzig, Inn Schneider B 2, fol. 91v. 
Ausgang des 17. Jahrhunderts ein Gehorsamsversprechen) ab. ${ }^{95}$ Unter den 30 Schneidersöhnen schwankt das Alter der iniurati zwischen 9 bzw. 10 und 22 Jahren. Das Gehorsamsversprechen leisteten sie dann im Alter zwischen 17 und 22 Jahren, wobei einzelne Abweichungen (Minimum 15 Jahre, Maximum 46 Jahre) existieren. Obwohl die Universitätsstatuten von 1620 das vollendete 17. Lebensjahr zur Vereidigung festschrieben, immatrikulierten sich (inklusive Gehorsamsversprechen) schon Burschen kurz vor ihrem 17. Geburtstag. Gottlieb Wilhelm Mieser, der Sohn des Vormeisters Johann Georg Wilhelm Mieser, schrieb sich sogar einige Wochen vor Vollendung seines 16. Lebensjahres ein. ${ }^{96}$

Mindestens acht der 30 Schneidersöhne erwarben hier in ihrer Heimatstadt den akademischen Grad magister artium, zwei von ihnen zudem den philosophischen Doktorgrad. Für sechs der nachgewiesenen Stipendienbewerber ist belegt, dass sie in Leipzig eine der ,höheren' Fakultäten besuchten (drei Jurisprudenz, drei Theologie). Christoph Wolle (1700-1761) brachte es 1746 sogar bis zum doctor theologiae. ${ }^{97}$ Einzelne der Stipendiaten bzw. Stipendienbewerber setzten ihre Studien an anderen Universitäten fort und erlangten dort universitäre Abschlüsse. So finden sich beispielsweise in den Jenenser und den Wittenberger Matrikeln entsprechende Einträge. ${ }^{98}$

Über den weiteren Werdegang der Bewerber um das Schneider-Stipendium konnte nur wenig eruiert werden, doch verweisen andere Untersuchungen auf den nicht ganz seltenen sozialen Aufstieg von Handwerkersöhnen durch ein Studium. ${ }^{99}$ Dabei war das Erlangen eines akademischen Abschlusses oder Grades nicht vorrangiges Ziel des Studiums, was die niedrige Zahl an Abschlüssen in den Matrikelbüchern erklären könnte. Vielmehr diente der Hochschulbesuch der persönlichen Weiterentwicklung und der Vorbereitung des späteren beruflichen Werdeganges.

95 Zur Unterscheidung der verschiedenen Kategorien an Universitätsverwandten und der Abschaffung des akademischen Eides sowie seiner Ersetzung durch ein Versprechen, die bisherigen Eidespflichten zu erfüllen, siehe ErLER, Matrikel I (wie Anm. 7), S. IL f.; Georg ERLer (Hg.), Die jüngere Matrikel der Universität Leipzig, 1559-1809. Als Personen- und Ortsregister bearbeitet und durch Nachträge aus den Promotionsakten ergänzt, Bd. II: Die Immatrikulationen vom Wintersemester 1634 bis zum Sommersemester 1709, Leipzig 1909, S. XXIX; ErLER, Matrikel III (wie Anm. 74), S. XI.

96 KA Leipzig, Taufbuch St. Nicolai 1698-1705, S. 269; ErLer, Matrikel III (wie Anm. 74), S. 266.

97 Erler, Matrikel III (wie Anm. 74), S. 466.

98 Fritz JunTke (Bearb.), Album Academiae Vitebergensis, Jüngere Reihe, Teil 3 (17101812) (Arbeiten aus der Universitäts- und Landesbibliothek Sachsen-Anhalt in Halle a. d. Saale 5), Halle a. d. Saale 1966, S. 71, 366, 428; Oтto KöHLER (Bearb.), Die Matrikel der Universität Jena, Bd. III: 1723 bis 1764, München u. a. 1992, S. 537.

99 Weitere Leipziger Beispiele hierzu aus dem 16. und 18. Jahrhundert führen an: KATRIN KeLLer, Zu materiellen Lebensverhältnissen kleiner gewerblicher Warenproduzenten am Beginn der Übergangsepoche vom Feudalismus zum Kapitalismus (Ende des 15. bis Anfang des 17. Jh.) - dargestellt am Beispiel von Leder- und Textilgewerben in Leipzig, Diss. phil. Leipzig 1987, S. 66 f.; LöfFler, Privatstipendien (wie Anm. 4), S. 349. 
Die weitere berufliche Entwicklung einzelner Stipendiaten (bzw. Stipendienbewerber) ließ sich dennoch verfolgen. So tauchte Gottlieb Wilhelm Mieser (1700-1755), der im sächsischen Pfarrerbuch fälschlicherweise als Schuhmachersohn identifiziert wurde, nach seinem Leipziger Studium 1723 als Substitut und 1725 als Pfarrer in Liptitz (westlich von Oschatz) auf. ${ }^{100}$ Johann Carl Haueisen, dessen Taufeintrag allerdings fehlt, wodurch seine Abstammung als Sohn des Schneidermeisters Abraham Haueisen nur durch das Handwerksbuch belegt ist, studierte Jurisprudenz, gründete frühzeitig eine Familie und wurde Antiquarius. ${ }^{101}$ Der bereits erwähnte Christoph Wolle, der sogar einen Eintrag in der Allgemeinen Deutschen Biographie erhielt, lehrte ab 1721 an der philosophischen Fakultät als Privatdozent, bevor er Anstellungen in verschiedenen Leipziger Kirchen, darunter St. Petri und St. Nicolai, erhielt. Über weitere Stationen stieg er bis zum Archidiakonus von St. Thomas (1741) und zum Theologieprofessor (1748) auf. ${ }^{102}$

\section{Die Bedeutung des Stipendiums}

Uneinigkeit herrscht in der Forschung bezüglich der Bedeutung von Studienstipendien für arme Studierende. Während z. B. Ulrike Denk aufgrund der Stipendienhöhe und der Vergabekriterien von einem geringen Anteil der durch Stipendien geförderten armen Studenten ausgeht, weist Thomas Adam in Bezug auf die Leipziger Universität (für das 19. Jahrhundert) einen beachtlichen Anteil an Stipendienbewerbern aus ärmeren Verhältnissen nach. Auch Hans-Ulrich Wehler vermutete einen größeren studentischen Anteil aus dem kleinbürgerlichen Milieu. Bernhard Ebneth betont bei dieser Frage die Notwendigkeit der Differenzierung nach Regionen, Fakultäten und Epochen. Übereinstimmung besteht dagegen in der Einschätzung, dass insbesondere Studenten aus den Familien der unteren Einkommensgruppen auf zusätzliche Einkommensquellen wie Stipendien angewiesen waren. ${ }^{103}$ Wie gezeigt wurde, kann im Falle des Johnschen Stipendiums diesbezüglich keine eindeutige Aussage getroffen werden. Es scheint weder eine durchgängige Bevorzugung materiell begünstigter Meisterfamilien noch eine ausgesprochene Unterstützung ärmerer Schneidersöhne gegeben zu haben. Für eine

100 Reinhold Grünberg (Bearb.), Sächsisches Pfarrerbuch. Die Parochien und Pfarrer der Ev.-luth. Landeskirche Sachsens (1539-1939), Teil II: Die Pfarrer der ev.-luth. Landeskirche Sachsens (1539-1939), Abteilung 2: M-Z, Freiberg 1940, S. 602.

101 KA Leipzig, Taufbuch St. Thomas 1781-1791, fol. 124v, Nr. 79; fol. 173v, Nr. 280; fol. 241v, Nr. 257; StadtA Leipzig, Inn Schneider B 11, fol. 28v.

102 Grünberg, Pfarrerbuch (wie Anm. 100), S. 1040; Paul Tschackert, Christoph Wolle, in: Allgemeine Deutsche Biographie 44 (1898), S. 548 f., Onlinefassung: http://www. deutsche-biographie.de.

103 Adam, Stipendienstiftungen (wie Anm. 9), S. 134, 140; Denk, Alltag (wie Anm. 5), S. 166 f.; Ebneth, Stipendium (wie Anm. 1), S. 516 f.; WeHler, Gesellschaftsgeschichte (wie Anm. 9), S. 298. 
abschließende Beantwortung dieser Frage wären weitere Auswertungen (besonders der Steuerbücher) notwendig.

Die Johnsche Stiftung war nicht ausschließlich für Studierende vorgesehen. Sollte sich kein geeigneter Kandidat finden, sollten die jährlichen Stiftungszinsen an arme Meister und Witwen der Zunft ausgeteilt werden. Ob und in welchem $\mathrm{Maß}$ von dieser Mittelverwendung Gebrauch gemacht wurde, konnte nicht belegt werden.

Von Bedeutung war die Stipendienstiftung darüber hinaus für ihre Administratoren und für die Schneiderinnung insgesamt. Wichtigen Einfluss auf die Vergabe des Stipendiums nahm die Zunftspitze. Zwar wurde über die Stipendienvergabe von der gesamten Zunft auf einer ihrer Versammlungen abgestimmt, doch sprachen viele Bewerber direkt bei den Ober- und Vormeistern vor, die die Angelegenheit schon im kleineren Kreis berieten oder den Kandidaten abweisen konnten. Auch bei den Abstimmungen vor der versammelten Meisterschaft hatte die Meinung der Handwerksältesten Gewicht und häufig folgten die auf 13 Tische verteilten Zunftmitglieder diesem Votum.

Das Stiftungskapital selbst kam der Schneiderinnung zugute. Sie durfte es in seinem Bestand nicht schmälern, wohl aber für das Handwerk vorteilhaft anlegen. Dies bedeutete, dass es beispielsweise für andere Vermögenswerte als Sicherheitsleistung fungieren konnte. Nachdem die Leipziger Schneider in verschiedenen Gasthöfen ihre Versammlungen abgehalten hatten, legten sie sich 1682 eine erste eigene Herberge zu. Sie erwarben für 2150 Gulden, darunter auch das Stiftungskapital, ein Haus auf dem Brühl im Norden der Stadt. Seitdem standen die 500 Reichstaler aus Michael Johns Erbe hypothekarisch auf diesem Gebäude, welches Zum Goldenen Beil genannt wurde. Der jeweilige Besitzer der Zunftherberge (anfangs die Innung selbst) war verpflichtet, die Stiftung zu bedienen, ${ }^{104}$ was bis ins 20. Jahrhundert hinein gelang.

\section{Fazit}

Am Beispiel einer fast gänzlich vergessenen privaten Stipendienstiftung wurde gezeigt, wie trotz problematischer Quellenbasis Einblicke in vergangene Denkund Lebenswelten gegeben werden können. Die vorgestellte Stipendienstiftung des Sekretärs und Schneidersohnes Michael John besaß eine einzigartige, langlebige Stiftungsstruktur, die in Leipzig und Sachsen trotz ihrer hohen Inanspruchnahme ohne Nachahmung blieb. Keine andere sächsische Stipendienstiftung vergab ihre Zuwendungen allein an die Nachkommen eines einzelnen Gewerbes. Der Wunsch im Milieu des kleinbürgerlichen Handwerks, die allgemeine Bildung zu heben und die Werkstattübernahme durch die Nachkommen zu ermöglichen, war verständlicherweise stärker. Damit weist diese Stiftung zugleich auf die Vielfalt der vormodernen Stiftungslandschaft hin.

104 StadtA Leipzig, Ratsbuch 145 (1696-1697), fol. 201r-202v; Germar, Schneiderhandwerk (wie Anm. 48), S. 91. 
Die Bedeutung des Johnschen Schneider-Stipendiums ist ambivalent zu beurteilen. Für die Zunft schufen die Kollatur und die Verwaltung der Stiftung neue Einflussräume. Die Aufnahme eines Studiums für ärmere Personen wurde lediglich in begrenztem Maße erleichtert, da die individuelle Bedürftigkeit als Zuteilungsgrund anscheinend nicht oder nur nachrangig berücksichtigt wurde und die Stipendienhöhe zumindest am Ende des 18. Jahrhunderts völlig unzureichend blieb. Zudem hatten die Bewerber jahrelang auf die Auszahlung der Stipendiengelder zu warten. Dennoch konnten auch mithilfe dieser Unterstützung viele Personen aus einem Leipziger Massenhandwerk studieren und ihren beruflichen und sozialen Aufstieg vorbereiten, denen dies sonst schwerer möglich gewesen wäre. Die Schneiderinnung, in deren Hände das Stiftungskapital gelegt wurde, trug erheblich zur Ausgestaltung der Stiftungswirklichkeit bei. Um die Inanspruchnahme rangen die potenziellen Stipendiaten und ihre Familien mitunter heftig mit der Innung und dem Stadtrat. Nicht allein unter diesem Aspekt gibt die Untersuchung historischer Stipendienstiftungen wichtige Hinweise über das Funktionieren sozialer Gemeinschaften.

\section{ANHANG}

Tab. 1 Biografische Angaben zu den Bewerbern des Johnschen SchneiderSTIPENDIUMS, Teil I

Erläuterungen: Die tabellarischen Angaben sind aufgrund der Überlieferungssituation mit Unsicherheiten behaftet. Es gilt insbesondere zu beachten: Zu Spalte 1) Unsicherheiten in dieser Spalte beziehen sich auf den recherchierten Vornamen. Alternative Schreibweisen des Familiennamens sind in Klammern angegeben. $\mathrm{Zu}$ Spalte 3) Enthaltene Abkürzungen im Matrikeleintrag sind nach ERLER, Matrikel I (wie Anm. 7) aufzulösen. Zu Spalte 4) Angabe des Alters bei Studienbeginn. Da sowohl der Taufbucheintrag nur einen etwaigen Anhaltspunkt für das genaue Geburtsdatum liefert, als auch die Matrikeleinträge mit Unsicherheiten behaftet sind, ist die Angabe des Alters bei Studienbeginn mit einer gewissen Unschärfe behaftet. Bei Angabe von zwei Zahlen verweist die erste auf das ungefähre Alter bei der Deposition, die zweite auf dasjenige zum Zeitpunkt des Gehorsamsversprechens. Zu Spalte 5) Angabe des Alters bei erstmaliger Stipendienbewerbung. $\mathrm{Da}$ Belege nur sporadisch vorhanden sind, ist nicht auszuschließen, dass in einzelnen Fällen eine Erstbewerbung erheblich früher erfolgte.

Quellen: KA Leipzig, Taufbücher St. Nicolai 1609-1615, 1677-1684, 1690-1697, 1698-1705, 1717-1722, 1760-1763, 1764-1771, 1772-1776, 1777-1780, 1781-1787, Taufbücher St. Thomas 1678-1691, 1692-1697, 1750-1754, 1755-1761, 1762-1770; StadtA Leipzig, II. Sektion I (F) 101, II. Sektion O (F) Nr. 92, Inn Schneider B 2, B 11 und C 14; Erler, Matrikel I (wie Anm. 7), Matrikel II (wie Anm. 95), Matrikel III (wie Anm. 74); Germar, Schneiderhandwerk (wie Anm. 48). 


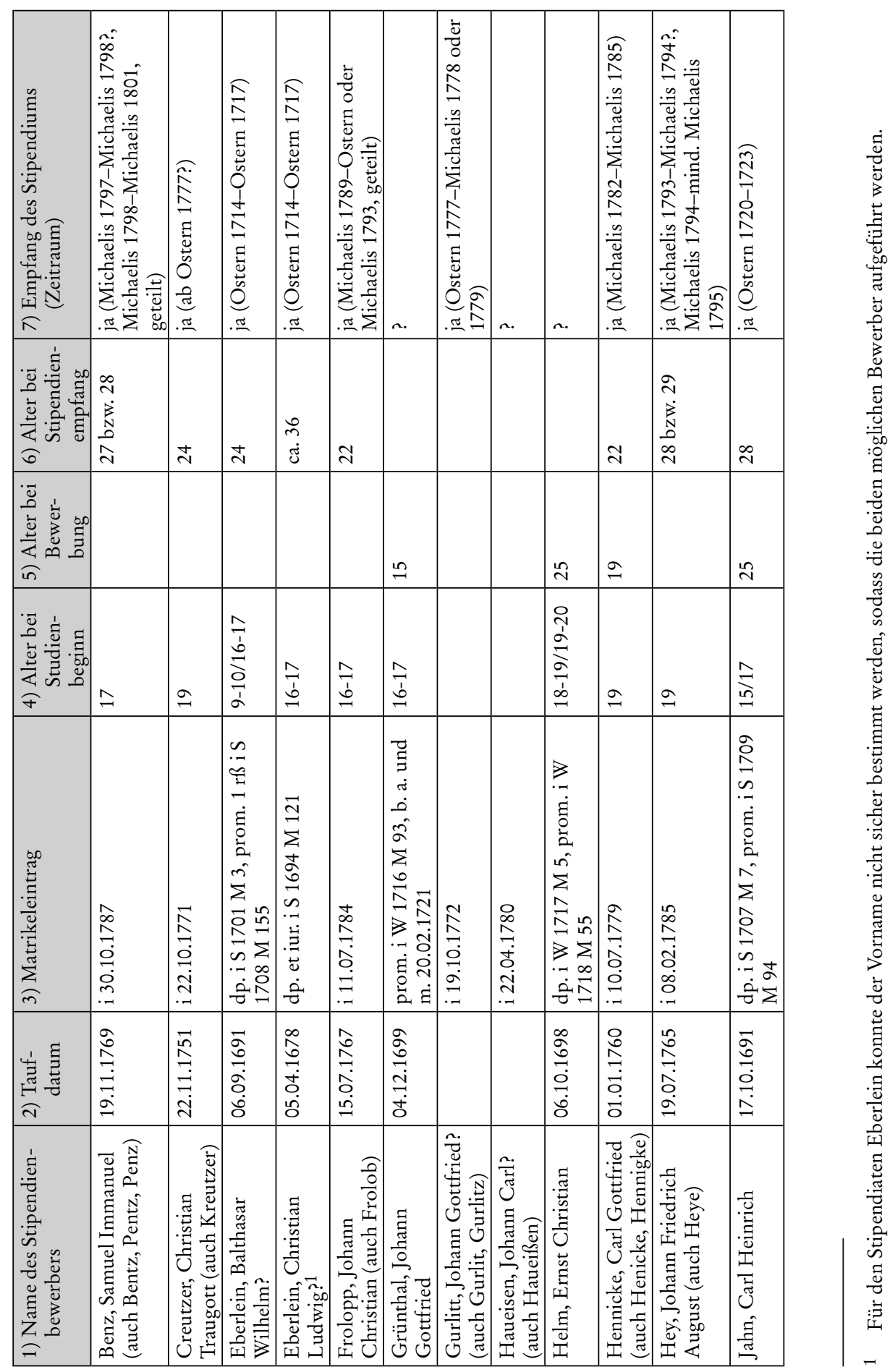




\begin{tabular}{|c|c|c|c|c|c|c|c|c|c|c|c|c|c|c|}
\hline 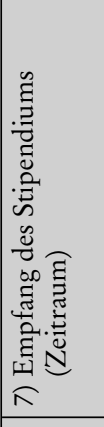 & n. & 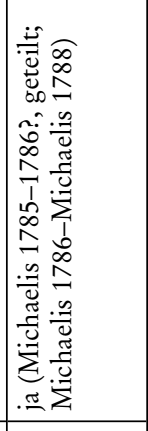 & n. & 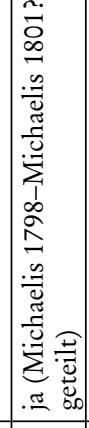 & & 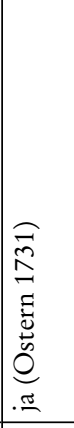 & n. & : & 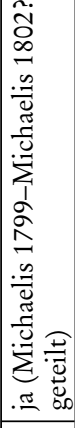 & 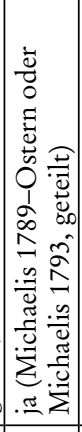 & & 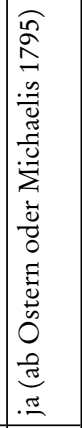 & 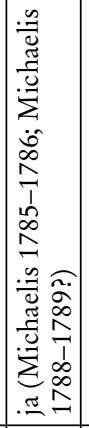 & \\
\hline 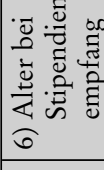 & & 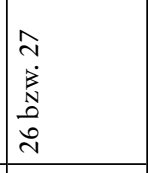 & & is & & ì & & & $\pi_{4}^{2}$ & $\stackrel{\infty}{\sim}$ & & i & $\bar{v}$ & \\
\hline 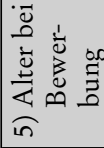 & & $=$ & $\tilde{m}$ & & & & \pm & $\tilde{\lambda}$ & & & $\approx$ & & & \\
\hline 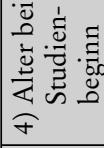 & $\approx$ & $\approx$ & 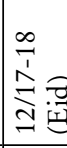 & $\nexists$ & & $\begin{array}{l}\text { a } \\
\text { a } \\
\end{array}$ & $\stackrel{\substack{n \\
\Xi}}{\Xi}$ & $\bar{\lambda}$ & $\lesssim$ & 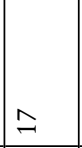 & $\stackrel{\infty}{\sim}$ & $\approx$ & $\begin{array}{l}\hat{1} \\
\hat{b}\end{array}$ & \\
\hline$\underset{\substack{\infty \\
\hdashline}}{\infty}$ & 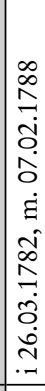 & 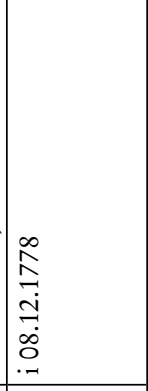 & 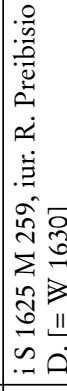 & 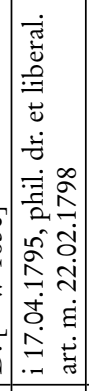 & 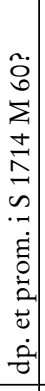 & 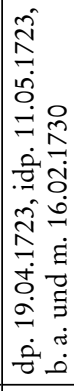 & 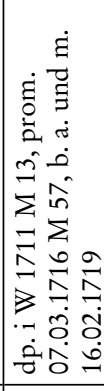 & 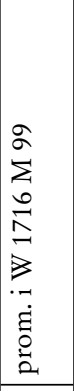 & 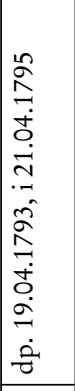 & 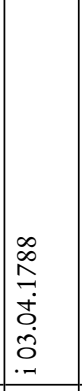 & 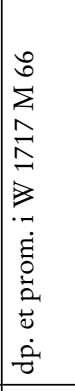 & 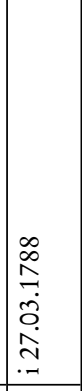 & 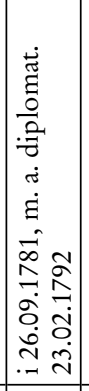 & $\begin{array}{l}20 \\
0 \\
7 \\
z \\
0\end{array}$ \\
\hline 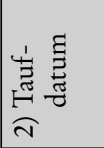 & 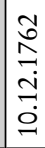 & 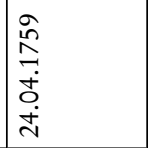 & 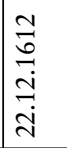 & $\begin{array}{l}\infty \\
\stackrel{1}{0} \\
\\
0 \\
0 \\
0 \\
0\end{array}$ & & 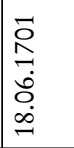 & $\mid$\begin{tabular}{c}
8 \\
\hdashline \\
\hdashline \\
\hdashline
\end{tabular} & 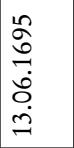 & 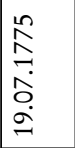 & 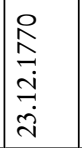 & 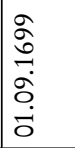 & 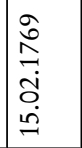 & 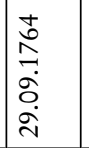 & $1 \infty$ \\
\hline 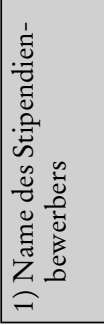 & 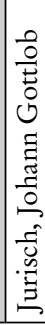 & 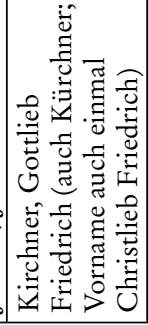 & 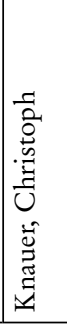 & 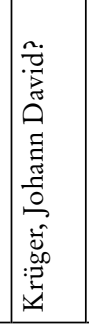 & & 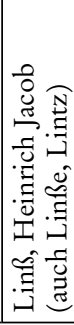 & 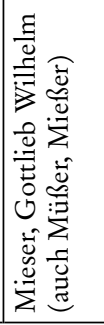 & 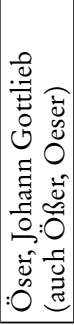 & 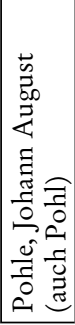 & 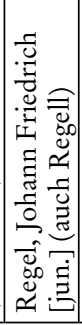 & 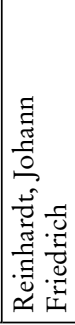 & 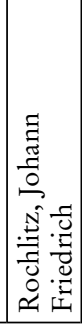 & 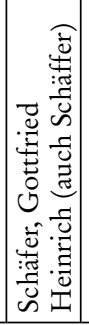 & 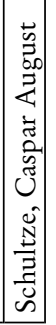 \\
\hline
\end{tabular}




\begin{tabular}{|c|c|c|c|c|c|}
\hline 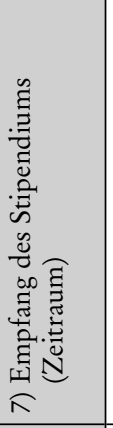 & : & 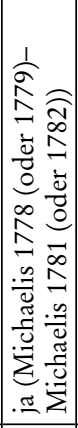 & 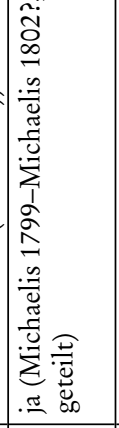 & 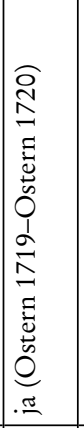 & $n$. \\
\hline 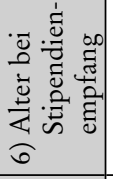 & & $\bar{\lambda}$ & $\stackrel{\infty}{\rightarrow}$ & $\stackrel{\infty}{\sim}$ & \\
\hline 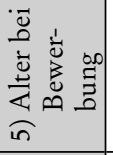 & in & 으 & & $\frac{\tilde{N}}{\stackrel{N}{v}}$ & $\bar{\imath}$ \\
\hline 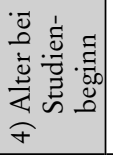 & fo & $\cong$ & $\underbrace{\partial}_{-}$ & 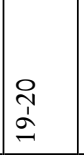 & $\underset{⿱ n}{\stackrel{\infty}{\sim}}$ \\
\hline 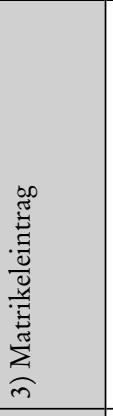 & 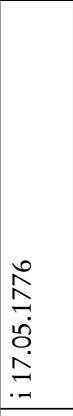 & 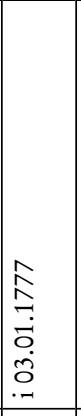 & 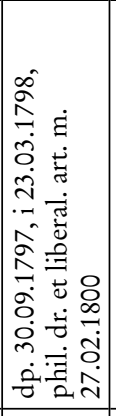 & 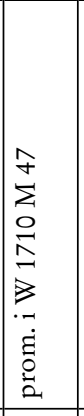 & 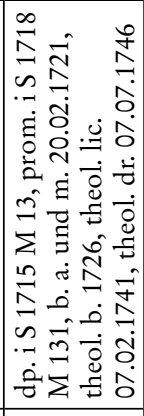 \\
\hline 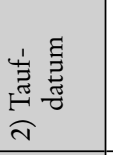 & 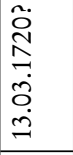 & $\begin{array}{l}\hat{n} \\
\stackrel{n}{\dot{1}} \\
\hat{0} \\
\dot{m} \\
\end{array}$ & 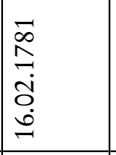 & 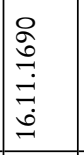 & 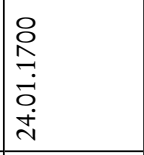 \\
\hline 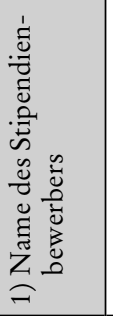 & 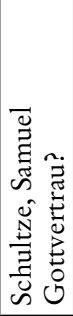 & 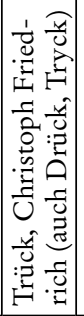 & 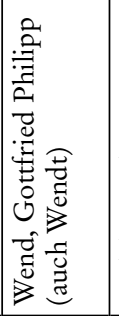 & 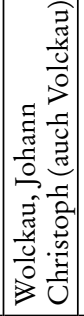 & 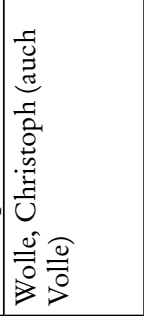 \\
\hline
\end{tabular}


Tab. 2 Biografische Angaben zu den Bewerbern des Johnschen SchneiderSTIPENDIUMS, TEIL 2

Erläuterungen: Zu Spalte 3) Wenn nicht anders angegeben, besaßen alle Väter das Leipziger Bürgerrecht und waren Mitglieder der Leipziger Schneiderinnung. Die Wohnangaben beziehen sich, wenn nicht anders angegeben, auf Leipzig. Zu Spalte 5) Weitere Quellenangaben siehe in Tabelle 1.

Quellen: KA Leipzig, Taufbücher St. Nicolai 1609-1615, 1690-1697, Taufbuch St. Thomas 1781-1791; StadtA Leipzig, II. Sektion I (F) 101, Inn Schneider B 2 und B 11, Polizeimeldebuch 1811-1813, Ratsleichenbücher Nr. 19 (1699-1707), Nr. 21 (1714-1720), Nr. 22 (1721-1727), Nr. 29 (1768-1779), Nr. 30 (1779-1784), Nr. 31 (1785-1793), Nr. 32 (1793-1799), Tit. XLII D (F) 153; ErLer, Matrikel III (wie Anm. 74); Germar, Schneiderhandwerk (wie Anm. 48); Grünberg, Pfarrerbuch II (wie Anm. 100); Fritz JuntKe (Bearb.), Album Academiae Vitebergensis, Jüngere Reihe, Teil 2 (1660-1710) (Arbeiten aus der Universitäts- und Landesbibliothek Sachsen-Anhalt in Halle a. d. Saale 1), Halle a. d. Saale 1952; Ders., Album 3 (wie Anm. 98); KöHLER, Jena (wie Anm. 98); Jürgen WinkLer (Bearb.), Kirchenbuch von Lützen 1770-1801 (Sterberegister) (Quelle 20b). Transkription aus der deutschen in die lateinische Schrift, Friedelsheim 2010; Tschackert, Wolle (wie Anm. 102). 


\begin{tabular}{|c|c|c|c|c|c|c|c|c|c|c|}
\hline 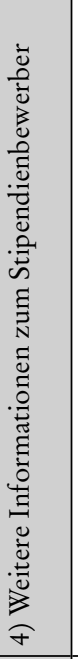 & & & & 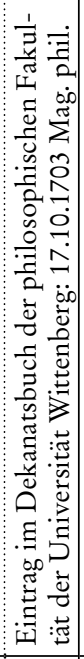 & & 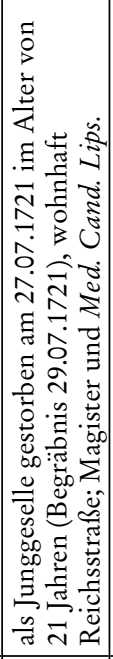 & & 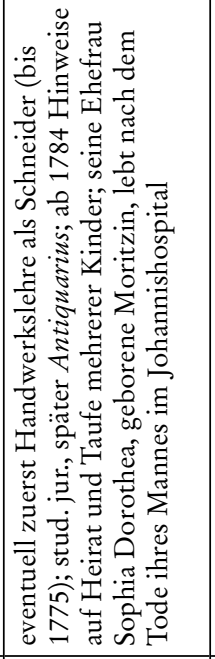 & & 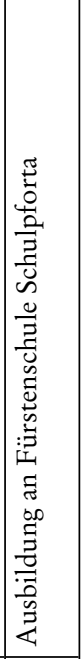 \\
\hline 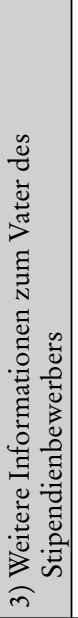 & 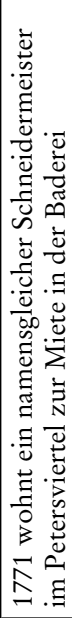 & & 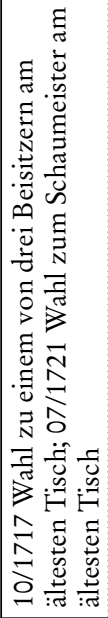 & & 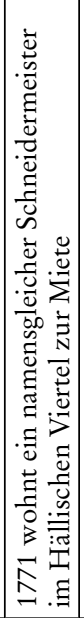 & 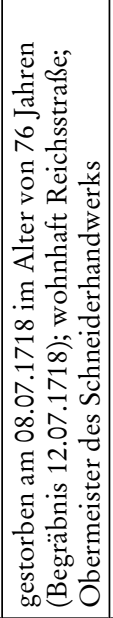 & 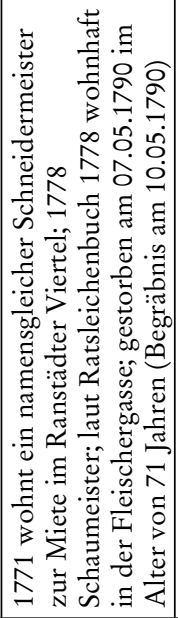 & 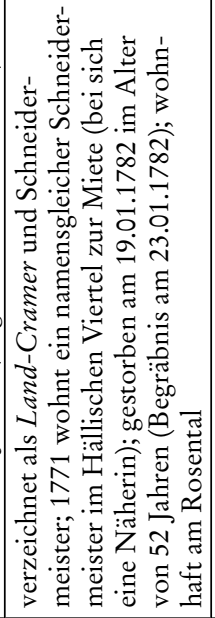 & & 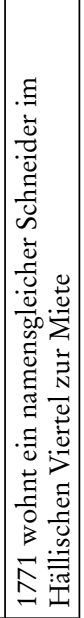 \\
\hline 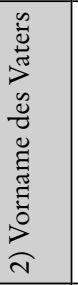 & 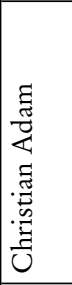 & 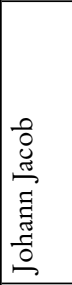 & 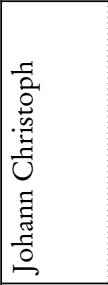 & 吾 & 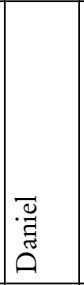 & 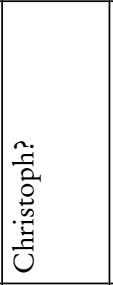 & 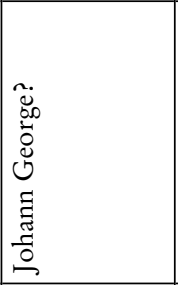 & 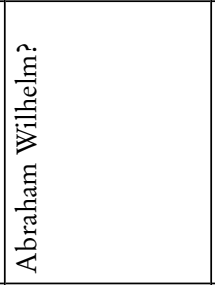 & 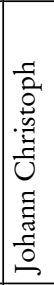 & 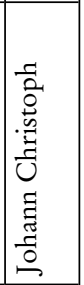 \\
\hline 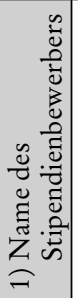 & 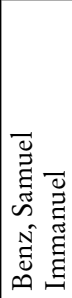 & 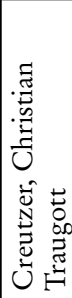 & 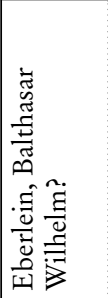 & 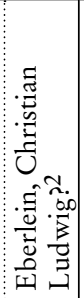 & 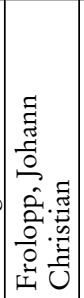 & 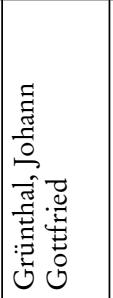 & 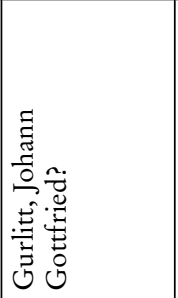 & 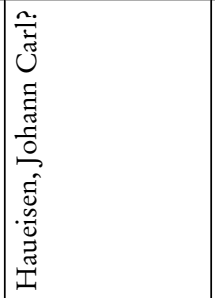 & 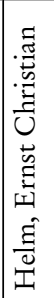 & 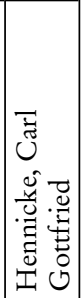 \\
\hline
\end{tabular}




\begin{tabular}{|c|c|c|c|c|c|c|c|c|c|}
\hline 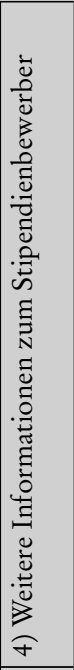 & & 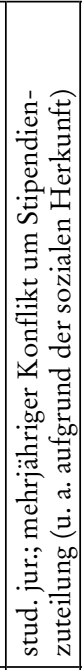 & & 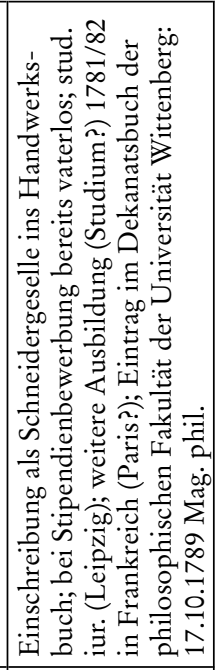 & 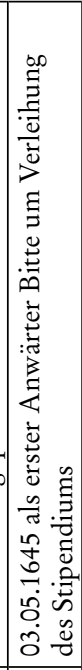 & 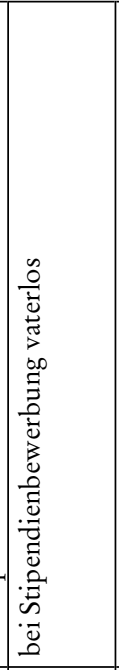 & 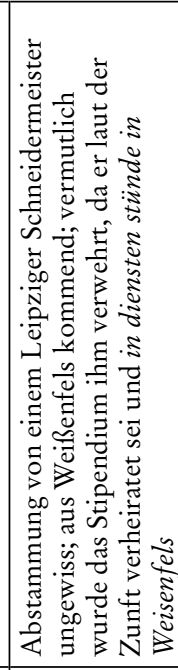 & 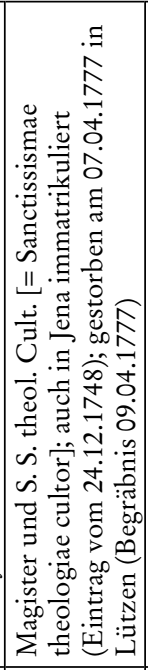 & 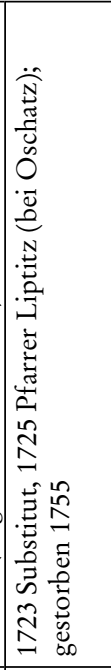 \\
\hline 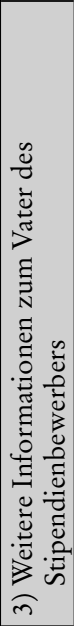 & 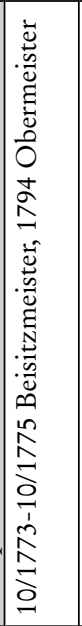 & 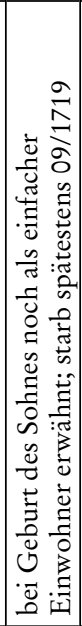 & 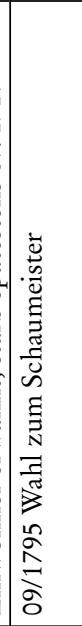 & 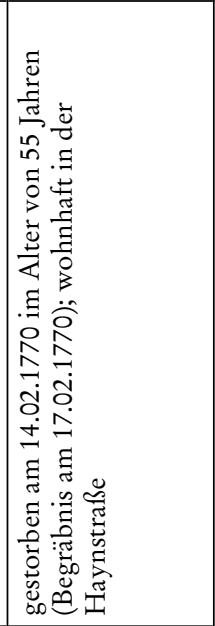 & & 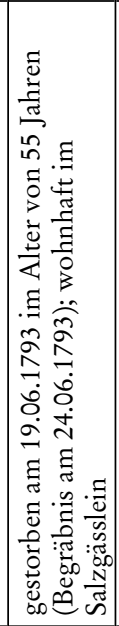 & & & 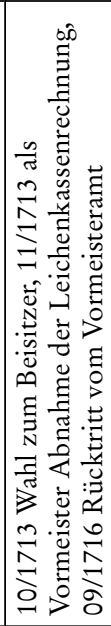 \\
\hline 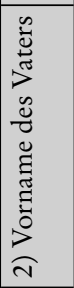 & 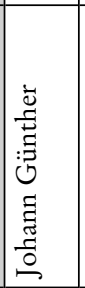 & 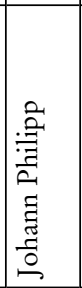 & 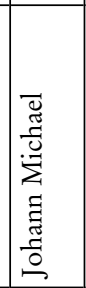 & 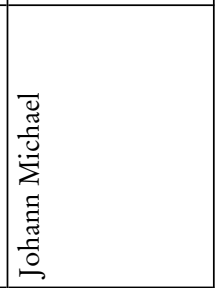 & 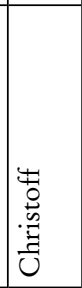 & 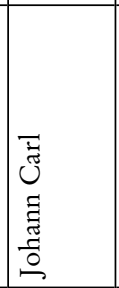 & n. & 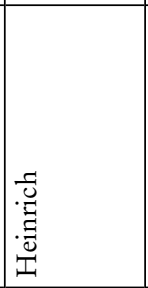 & 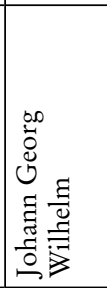 \\
\hline 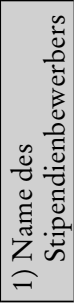 & 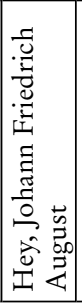 & 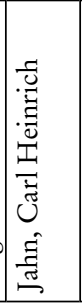 & 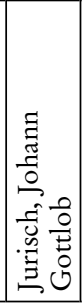 & 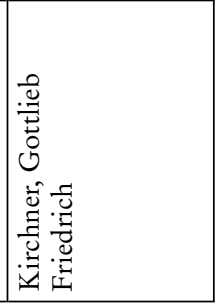 & 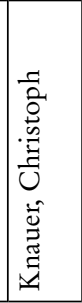 & 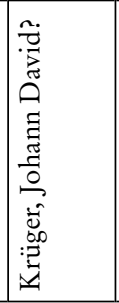 & 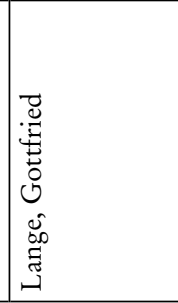 & 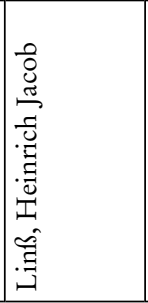 & 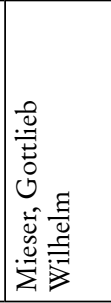 \\
\hline
\end{tabular}




\begin{tabular}{|c|c|c|c|c|c|c|}
\hline 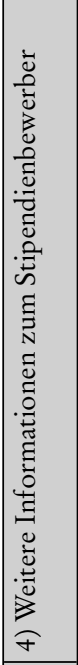 & 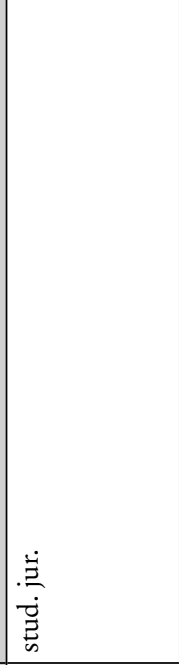 & 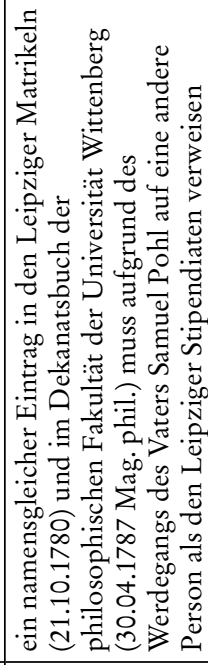 & 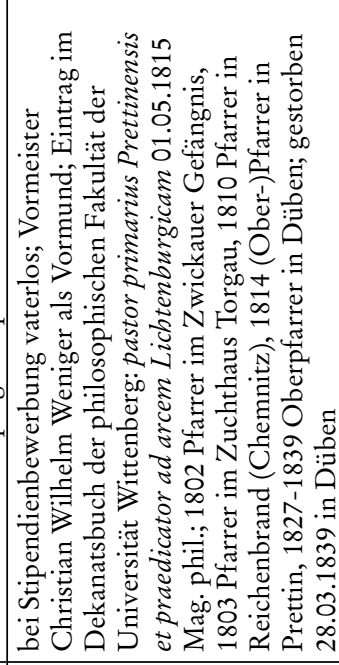 & & & \\
\hline 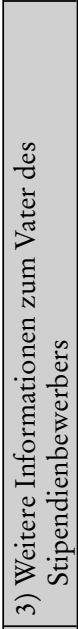 & 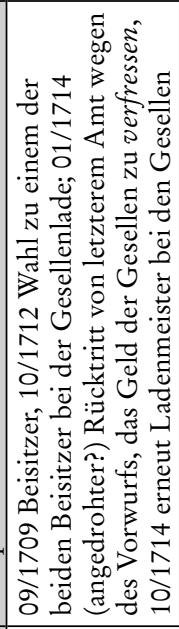 & 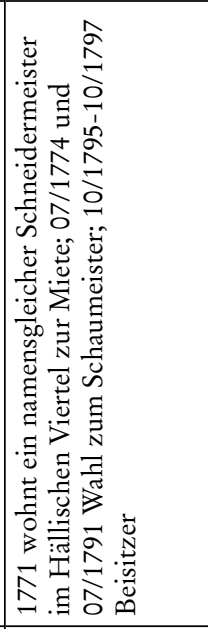 & 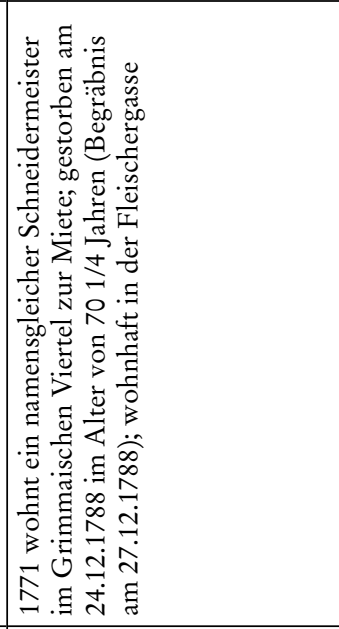 & & 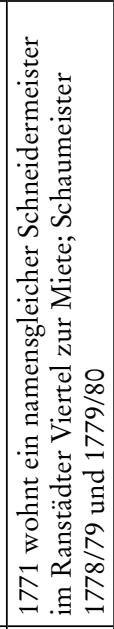 & 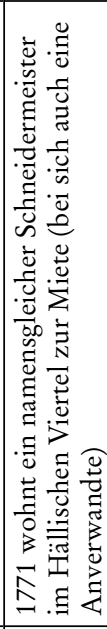 \\
\hline 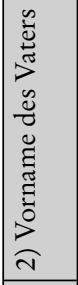 & 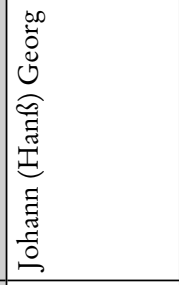 & $\begin{array}{l}\overrightarrow{\tilde{D}} \\
\overrightarrow{\tilde{g}} \\
\tilde{n}\end{array}$ & 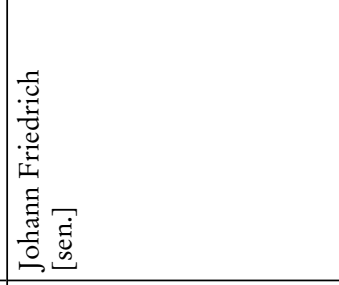 & 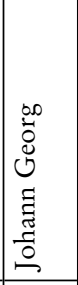 & 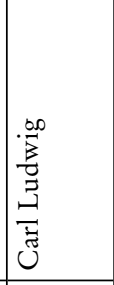 & 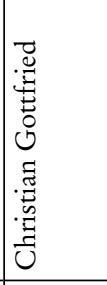 \\
\hline 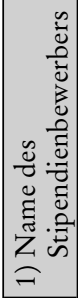 & 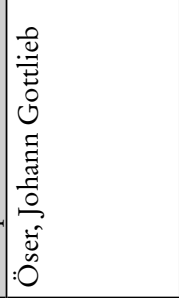 & 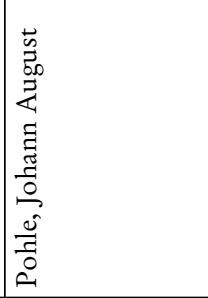 & 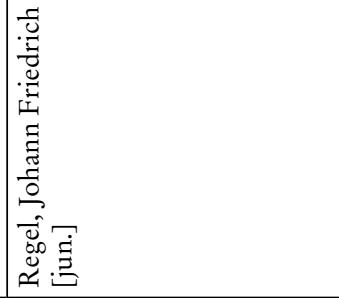 & 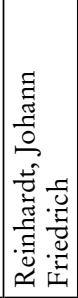 & 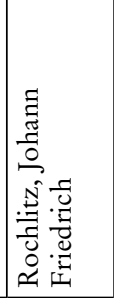 & 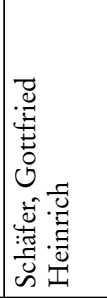 \\
\hline
\end{tabular}




\begin{tabular}{|c|c|c|c|c|c|c|}
\hline 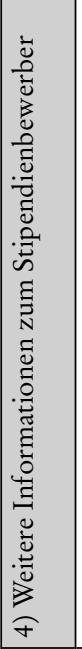 & 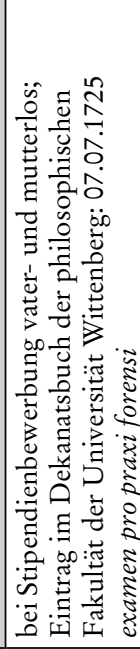 & 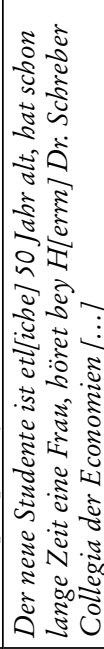 & & & & 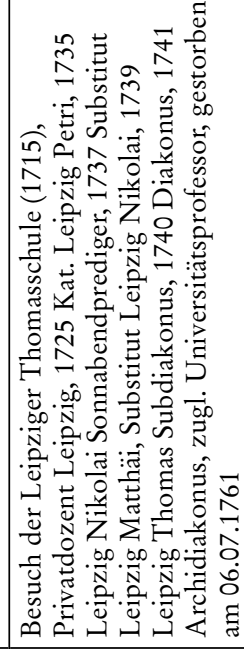 \\
\hline 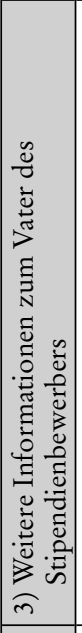 & 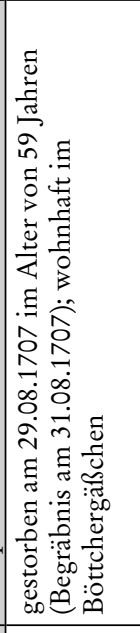 & & 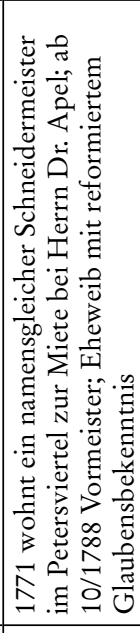 & 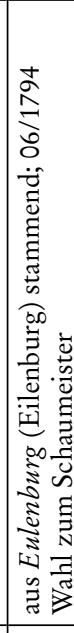 & 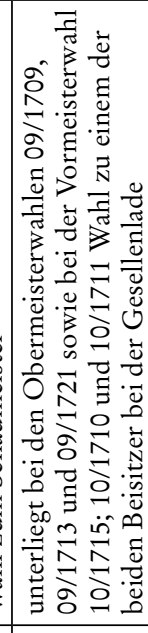 & \\
\hline 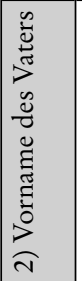 & 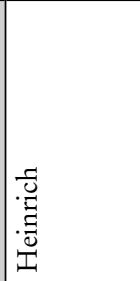 & 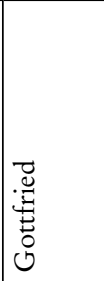 & 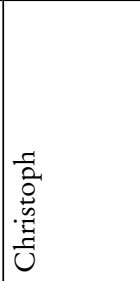 & 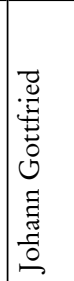 & 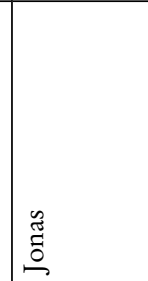 & 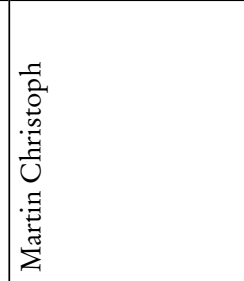 \\
\hline 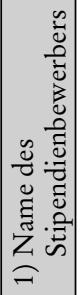 & 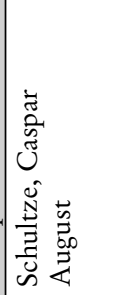 & 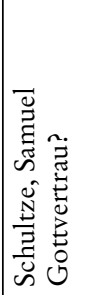 & 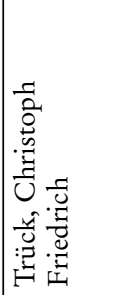 & 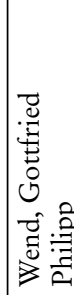 & 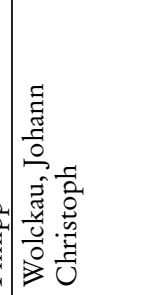 & 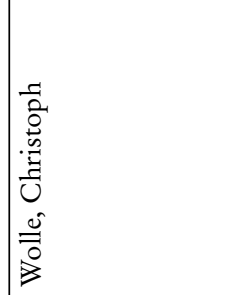 \\
\hline
\end{tabular}

